\title{
Ground-to-GEO optical feeder links for very high throughput satellite networks: Accent on diversity techniques
}

\author{
Alberto Mengali $^{1}$ ( ) | Charilaos I. Kourogiorgas ${ }^{2}$ | Nikolaos K. Lyras ${ }^{2}$ | \\ Bhavani Shankar Mysore Rama Rao ${ }^{1}$ [1] | Farbod Kayhan ${ }^{1}$ [ ] | \\ Athanasios D. Panagopoulos ${ }^{2}$ (1) | Thomas Bäumer ${ }^{3}$ | Konstantinos Liolis ${ }^{3}$
}

\author{
${ }^{1}$ Interdisciplinary Centre for Security, \\ Reliability and Trust (SnT), University of \\ Luxembourg, Luxembourg, Luxembourg \\ ${ }^{2}$ School of Electrical and Computer \\ Engineering, National Technical University of \\ Athens, Athens-Zografou, Greece \\ ${ }^{3}$ SES S.A., Technology Department, Château \\ de Betzdorf, Betzdorf, L-6815, Luxembourg
}

\section{Correspondence}

Bhavani Shankar Mysore R, 29 Avenue J. F. Kennedy, L-1855 Luxembourg.

Email: Bhavani.Shankar@uni.lu

\section{Funding information}

National Research Fund, Luxembourg, Grant/ Award Number: 10064089; Government of Luxembourg (Ministry of Economy), Grant/ Award Number: 40000113462/15/NL/NDe

\begin{abstract}
Summary
This paper studies the use of optical feeder links in very high throughput satellites (VHTS) networks with emphasis on gateway diversity techniques to mitigate the inherent propagation losses in optical frequencies. Focusing on a GEO scenario, the paper considers a system-wide approach investigating various challenges of optical feeder links. These include transmission schemes amenable for transparent on-board processing, optical channel models taking into account blockage by clouds and fading caused by atmospheric turbulence in addition to complexity of on-board and on-ground processing. The channel models are then used to dimension the ground segment towards ensuring a given availability percentage (e.g., 99.9\%). The channel model and payload complexity further influence the choice of link layer techniques used for counteracting fading due to atmospheric turbulence in the absence of blockage. An elaborate end-to-end simulator incorporating the proposed channel models capturing the nuances of various processing blocks like optical-electrical conversion is developed. The system performance results provide interesting insights and a framework for assessing the feasibility and advantages of optical feeder links in VHTS systems.
\end{abstract}

\section{KEYWORDS}

analog and digital transparent payloads, channel models, GEO networks, microdiversity and macrodiversity, optical feeder links, optical ground station network selection, optical received time series, optical SNR

\section{1 | INTRODUCTION}

A key challenge towards future generation very high throughput satellites (VHTS) is the limited spectrum of about $2 \mathrm{GHz}$ available in the Ka band $(20 / 30 \mathrm{GHz})$. A potential solution for resolving this issue could be to move the feeder links from the Ka band to the Q/V band (40/50 GHz) ${ }^{1-4}$ or even to the $\mathrm{W}$ band $(70 / 80 \mathrm{GHz})^{5}$ where bandwidths of up to $5 \mathrm{GHz}$ are available. While this allows for the use of radio frequency (RF) domain expertise, the bandwidth available is limited compared with requirement estimates and the spectrum is also solicited in the emerging $5 \mathrm{G}$ paradigm. ${ }^{6}$ Another revolutionary solution is to move the feeder link from RF frequencies to optical frequencies. ${ }^{7-9}$ Both these approaches are challenging due to the attenuation by atmospheric phenomena (e.g., rain and clouds) whose severity increases with the frequency. In both cases, a network of multiple gateways with smart switching capabilities is thus envisaged. ${ }^{10,11}$ While RF at high frequencies is less impaired than the optical counterpart, larger number of gateways is nonetheless required to achieve very high throughputs due to the limited available bandwidth per 
gateway (100-1000 times lower) compared with optical frequencies. ${ }^{10}$ This translates into a significant escalation in the cost of the ground-segment, favoring the deployment of feeder links in optical frequencies. Use of free space optics (FSO) communications also has additional advantages with respect to the use of RF as (a) absence of frequency regulation constraints due to the highly directive antennas, (b) smaller systems with potentially lower power consumption, (c) enhanced security due to directivity of the beams, and (d) ability to completely reallocate RF frequency bands to the user link.

While optical links offer a variety of advantages, they are impaired by several atmospheric phenomena like clouds, aerosols, turbulence, etc. Among these impairments, cloud coverage constitutes the predominant fading mechanism, resulting to the blockage of the link. ${ }^{12}$ Critical to the evaluation of optical satellite communication systems is the probability that the link is not blocked by clouds, also termed as cloud-free line Of sight (CFLOS) probability. In the literature, several methodologies have been proposed for the computation of CFLOS probability ${ }^{13-16}$ taking into account the spatial and temporal variability of clouds. Nevertheless, even under CFLOS conditions, the optical systems are severely affected due to propagation of optical waves through atmosphere. The phenomenon of atmospheric turbulence has significant impact on the signal under CFLOS conditions necessitating its accurate estimation in the link budget analysis ${ }^{17}$ taking also into account the beam wander, beam spread, and amplitude scintillation. In the literature, several studies have been conducted for the evaluation of this phenomenon. ${ }^{18-21}$ Moreover, in addition turbulence, the molecules which are present in the atmosphere, the cirrus clouds which are shaped from ice crystals, and the aerosols with size comparable with the wavelength of the signal, cause the reduction of signal amplitude. ${ }^{22}$

Several techniques have been proposed to mitigate the propagation impairments towards envisaging a feasible FSO system. For cloud coverage, macrodiversity technique is employed where multiple optical ground stations (OGSs) constituting an optical ground station network (OGSN) are employed. ${ }^{13,23,24}$ These stations are placed at cloud free areas with separation distance of order of hundreds of kilometers, so as the desired CFLOS probability of the whole network is achieved. ${ }^{14,25}$ One of the main drawbacks of this approach is the cost of having several ground stations. On the other hand, mitigation techniques for turbulence depend on the uplink or downlink scenario. The use of various diversity techniques as mitigation to turbulence effects will be termed as microscopic diversity techniques. Focusing on the optical feeder uplink, transmitter diversity, ${ }^{26}$ wherein multiple apertures are placed at a distance longer than the coherence length of turbulence, is used to combat turbulence. Further, many works have been focused on exploiting the diversity gain achievable from multiple input multiple output (MIMO) optical setups, mostly for terrestrial FSO networks. Among the spatial diversity methods that have received particular interest, we can mention: optical spatial modulation (OSM), ${ }^{27,28}$ repetition coding (RC), ${ }^{7,26,29-32}$ and optical space time codes (OSTC). ${ }^{33-37}$

However, all of these works assume a constructive combination of the transmitted beams at the receiver. Different optical paths, separated by at least the coherence length of turbulence (which is usually smaller than a few tenths of metre), propagate through media having different characteristics; hence, different optical waves are received with a different amplitude and phase at the receiver. While constructive combination is a reasonable assumption when dealing with incoherent sources of light (e.g., LEDs) common in terrestrial networks, this is not always valid when dealing with coherent light sources (i.e., lasers). Laser beams at the same frequency may combine destructively, leading to insufficient light reaching the receiving photodetector. The impact of random phase combination of the optical beams over lognormal channels has been analyzed in Mengali et al. ${ }^{38}$ where the noncoherent combination completely negates the benefits of RC. For this reason, most of the works analyzing spatial diversity in satellite optical feeder links assume frequency/polarization separation in addition to the spatial one in order to always achieve nondestructive combination. ${ }^{26,31,32}$

This paper focuses on the investigation of optical feeder link techniques for future generation GEO VHTS systems considering their impact both on the ground segment and space segment. We restrict ourselves to transparent payloads ${ }^{10}$ to avoid significant payload complexity while allowing it to be future-proof; the user link is assumed to be in Ka band. This hybrid architecture seems to be the feasible way forward for broadband satellite systems serving mass market in the RF regime. Because a transparent payload is used, quality of the downlink transmission is inherently impacted by the uplink transmission. Hence, the elements characterizing the uplink transmissions like optical to electrical conversion (and vice-versa), optical channel, RF, and optical modulation/ demodulation impact signal-to-noise-ratio (SNR) on the downlink. As a result, the two links are coupled and cannot be separated; this motivates an end-to-end analysis. Within this framework, the contributions of this paper are as follows:

- Providing a methodology for the dimensioning of OGSN based on a CFLOS time series generator, considering also the impact of the OGS altitude

- Employing a consolidated channel model for the generation of received power time series using stochastic differential equations (SDEs) for the uplink scenario

- Exploitation of microdiversity to achieve flexibility and efficiency in the deployment of a hybrid optical/RF communication system; in particular, a systematic study of microdiversity including the assessment of impact of channel phase is undertaken

- Evaluation of the OGSN performance taking into account not only the outage due to blockage but also due to the microscopic fading, an exercise not considered earlier, leading to an improved system dimensioning exercise

- Evaluation of the end-to-end frame error rate (FER) performance of a hybrid architecture with optical feeder uplink and RF user downlink. To the best of the authors knowledge, it is the first time a study is done taking into account the various impairments including 
the inefficiencies of FSO modulation, various noise components in the FSO demodulation, ${ }^{39}$ satellite transponder impairments, and user link artifacts.

The outcomes from the paper provide for a first assessment of the optical feeder link with transparent payloads that enables its performance comparison vis-a-vis RF systems, thereby impacting future VHTS architectures.

The rest of this paper is organized as follows. In Section 2, scenario and system model describing the GEO network and the different system architectures are described. In Section 3, the channel model considered in this study is detailed including a selection of the OGSN sites. Subsequently, in Section 4, the microscopic and macroscopic diversity techniques are highlighted. In Section 5, the simulation environment, the parameters, and end-to-end performance are presented. Finally, the outcomes are summarized in Section 6.

\section{2 | SCENARIO AND SYSTEM MODEL}

In this section, we describe the application scenario of optical feeder links and model the various system components including the transmission schemes, payload, and user terminal (UT) processing.

\subsection{Scenario}

The GEO optical feeder link scenario, illustrated in Figure 1, proposes a hybrid system employing optical feeder links to the GEO satellite for a typical broadband application involving DVB-S2x framing. At the OGS, a baseband DVB-S2x signal is suitably converted to the optical domain and transmitted using a laser to the transparent GEO satellite. On-board the satellite, the received optical signal is down-converted to the electrical domain, amplified and forwarded to the UTs in RF band (specifically, Ka band). The key components in the scenario are detailed below. Note that the system scenario was defined within the ONSET project by SES being in line with its business interests. ${ }^{40}$

\subsubsection{Space segment}

A multibeam VHTS generating several tens of beams to achieve high transmission and reception gains towards the UTs distributed across its service area is considered. A pan-European coverage is assumed and multibeam architecture allows for the implementation of frequency reuse; typically, a four-color scheme resulting in a frequency reuse factor of 20 is adopted in such multibeam satellite network.

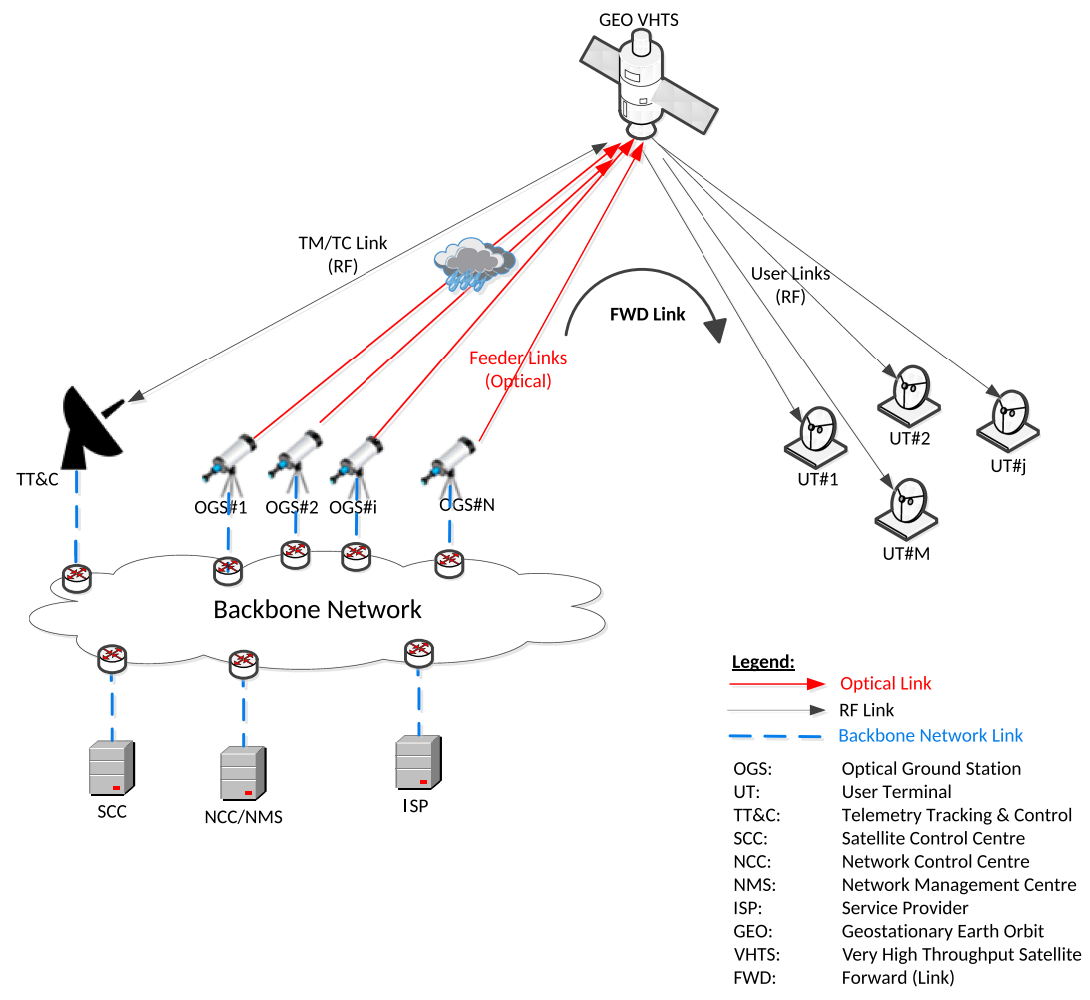

FIGURE 1 Scenario description [Colour figure can be viewed at wileyonlinelibrary.com] 
A transparent payload is considered where the uplink optical signal is converted into RF domain for downlink without demodulating the underlying DVB-S2x signal. Further details on the optical-electrical conversion are detailed in Section 2.2. The on-board processing involves channelization and amplification using a standard transponder comprising input multiplexing (IMUX) filter, a high power amplifier (HPA), and an output multiplexing (OMUX) filter. The transparent architecture benefits from lower complexity and will be agnostic to evolving RF air-interface formats.

\section{\begin{tabular}{l|l}
2.1 .2 & User segment
\end{tabular}}

The user segment is composed of a set of fixed (stationary) UTs operating in Ka band which are assumed to be DVB-S2x compliant. The size of the terminal dish is typically $75 \mathrm{~cm}$, whereas its power ranges from 2 to $4 \mathrm{~W}$. As a baseline, we assume that with the feeder link being moved to the optical band, the spectrum released from the RF feeder link can be allocated to the user links. The scenario envisages no change to the existing user links which will continue to operate in the RF band (Ka band) where low cost UTs are already available. Thus, the cost of the optical feeder link in the long term can be potentially negligible compared to the revenue generating RF user links.

\subsection{3 | Ground segment}

In a simplistic set-up, each OGS consists of three main elements: a dome to protect the optical devices from rain, ice, and heavy wind; a telescope with a mirror for determining the gain of the optical signal; and an instrument box. The latter has two functions: modulating the signal received from the backbone network into an optical signal and controlling the fine pointing and tracking procedure of the telescope. To avoid excessively long paths through the atmosphere, we assume that the elevation angle will always be at least $30^{\circ}$. Despite the fact that the envisaged throughput of an OGS is sufficient to transmit the entire feeder link signal, multiple OGSs are considered to overcome the detrimental effects of clouds coverage. Frequent switching between gateways in a macrodiversity scheme is envisaged due to dynamic cloud formations, thereby necessitating an OGSN where the multiple CFLOS are interconnected through the Backbone Network.

To ensure the efficient management of the OGSN, the following elements are considered: (a) network control center (NCC) controls and synchronizes the OGSN and can potentially add some delay to the signal in order to take into account the different signal run times both within the ground network and from the OGS satellite; (b) network management center (NMC) monitors and schedules the link both within the OGSN and between the active transmitting OGS and the satellite. It controls the status of every single OGS and observes the weather data to estimate the availability of each station. A weather forecast algorithm is constantly recalculating, based on the on-site measurements and on public available data, the weather forecast of the different sites. In case of an expected link interruption (e.g., clouds and storm) or worse atmospheric conditions, the NMC starts to establish and synchronize a new link via the OGS with the best weather forecast; (c) satellite control center (SCC) is responsible for monitoring and controlling the functionality and orbit of the space segment; (d) telemetry tracking and control (TT\&C) is mandated to transmit and receive telecommand/telemetry (TC/TM) data to/from the space segment through an RF link. In addition, it will communicate to both the satellite to establish the optical link to the suitable OGS.

\section{2 | System model}

The system model for the aforementioned scenario depicting the forward link from the OGS through the GEO transparent satellite to the UT is described in Figure 2. The baseband data processing at OGS involves standard DVB-S2x modules including coding, modulation,

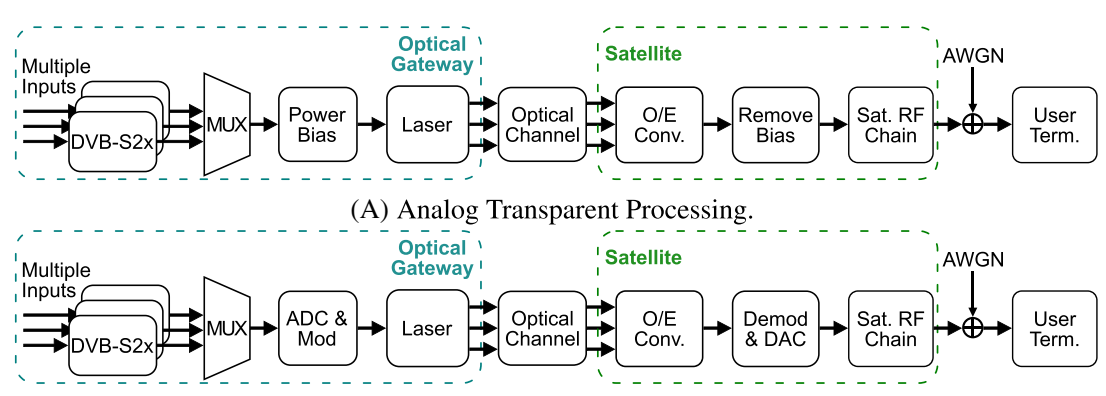

(B) Digital Transparent Processing.
FIGURE 2 System model [Colour figure can be viewed at wileyonlinelibrary.com] 
interleaving, framing, and baseband filtering; the interested reader can refer to previous study ${ }^{41}$ for additional details. Multitude of these DVB-S2x streams are then modulated into the optical domain; a VHTS will require a number of optical modulators (each operating at a different wavelength) to support traffic in the order of terabit/s. This is due to the bandwidth limitation of the components rather than spectrum availability in optical frequency. Photoreceptors are commercially available at up to $100 \mathrm{GHz}$ bandwidth at the moment, ${ }^{42}$ whereas front-end electronic have even lower speeds ${ }^{43}$ limiting the effective bandwidth per optical chain. In this work, like in Roy et al., ${ }^{32}$ we have considered an RF signal bandwidth of $1.5 \mathrm{GHz}$ before optical conversion, corresponding to roughly three DVB-S2x streams of $450 \mathrm{MHz}$ plus guard bands. Towards ensuring transparent solutions, two schemes are usually considered in the literature: Digital and Analog ${ }^{10}$ which are now briefly described.

\subsection{1 | Analog transparent scheme}

In this scheme, the users' signals from the DVB-S2x chain ${ }^{44}$ are aggregated and the resulting signal is used to modulate the intensity of a laser source. To reduce the distortion on the resulting light signal, direct modulation of the laser amplitude is not desired and external modulation, like the Dual Drive Mach-Zehnder Modulator (DD-MZM) for complex signals, is preferred. ${ }^{45}$ In this work, for simplicity, we assume a linear laser amplitude modulation, that is, the amplitude of the light at the output is an undistorted replica of the amplitude of the RF signal at its input. Subcarrier intensity modulation (SIM) is typically used in such systems and the same has been studied extensively in previous studies. ${ }^{46-49}$ In SIM, an electrical bias needs to be added to the aggregate signal to ensure its nonnegativity. This bias will be removed after opto-electrical conversion on board the satellite to restore the original RF signal, thus reducing the power efficiency of the system. Further, on-board the satellite, the received signal is first optically preamplified by means of a low noise optical amplifier and is then converted by a square law opto-electrical detector to recover the original DVB-S2x signals. After this process, the signals are processed using typical RF transponders before being downlinked to the users as in existing RF systems.

As will be discussed in Section 5.3, the power bias will cause an increase in the noise generated during the opto-electrical conversion process. The main advantage of this scheme is its rather simple implementation and low cost, both on the ground and space segment. The disadvantages are the lower useful signal power after the opto-electrical conversion and the inability to include digital fade-mitigation techniques at the transmitter. The first makes the scheme very sensitive to turbulence-fading, and the second limits fade mitigation techniques to transmitter diversity at the OGS and automatic gain control (AGC) functionalities on-board the satellite.

\subsection{2 | Digital transparent scheme}

In this scheme, the DVB-S2x aggregate signal is digitized by an analog-to-digital converter (ADC) at the OGS and the digitized samples are transmitted over the optical feeder link using an appropriate multilevel optical modulation format. While several modulation schemes are proposed in the literature to transmit the digitized signal, ${ }^{45}$ we focus on the on-off keying (OOK) modulation scheme given its simplicity. Similar to the analog case, the received signal is first optically preamplified by means of a low noise optical amplifier, converted by a square law opto-electrical detector and demodulated to obtain the digitized RF waveform. Subsequently, a digital-to-analog converter (DAC) is used to generate the analog waveform on-board of the satellite. The digital nature of the transmission allows incorporation of forward error correction (FEC) on the digitized bits as an additional way to mitigate the turbulence induced fading. Further, the scheme has the flexibility to incorporate new optical modulation and coding techniques. However, the system is more complex than analog transparent scheme (ATS) due to the requirement of high speed DAC and ADC. Furthermore, it offers relatively low spectrum efficiency in the optical channel, due to the required quantization, unless high order optical modulation are used.

\subsection{3 | Satellite: optical to electrical conversion}

The process of converting the optical received signal to electrical domain for transmission over the RF user link follows the steps illustrated in Figure 3A. We consider only one receiving antenna on-board the satellite to limit the payload mass and complexity. The signal light, coming from multiple colocated transmitters if microdiversity is employed and corrupted by the relative intensity noise (RIN) generated at the laser sources, is combined on the single-receiving aperture together with the light coming from background radiation sources (e.g., celestial bodies). Due to free space loss (FSL), the received optical signal power is very low and needs to be amplified, in our case using an erbium-doped fiber amplifier (EDFA). An interfering optical wave is generated by the spontaneous emission of photons inside the optical amplifier when the temperature is above $0^{\circ} \mathrm{K}^{39}$ This unwanted signal is also amplified while passing through the EDFA and translates into a significant noise term called amplified stimulated emission (ASE) noise. 


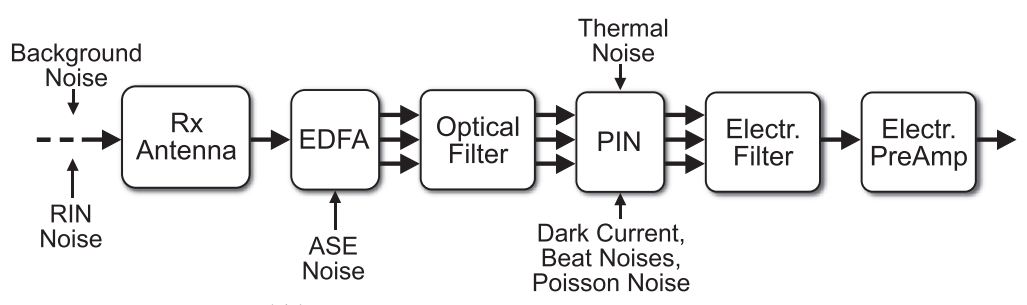

FIGURE 3 Detailed view of satellite and optical to electrical conversion blocks

(A) Optical to Electrical Conversion

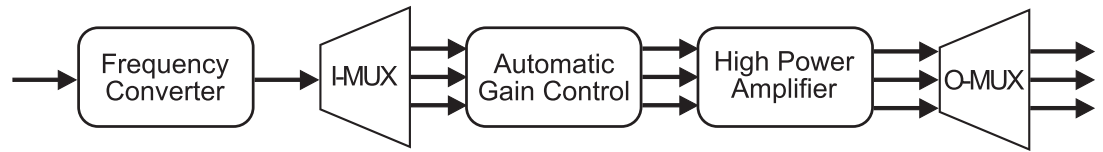

(B) Satellite Radio Frequency Chain.

After preamplification, the different beams arising from the transmitter microdiversity undergo separate processing before being recombined in the electrical domain. First, a bank of optical filters, whose bandwidth should match that of the signal being processed, is used to reduce the effect of the ASE noise. Because it is technologically challenging to create narrow-band optical filters, we assumed the minimum allowed bandwidth of the optical filters to be equal to the minimum spacing of the ITU dense wavelength division multiplexing (DWDM) frequency grid report ${ }^{44}$ (i.e., $12.5 \mathrm{GHz}$ ). After the optical filtering, the light beam reaches the area of the PIN photodiode that act as a mixer, down-converting the signal back the electrical domain. As a consequence of concurrent presence of ASE noise and useful signal, some beat noise terms are produced during the frequency down-conversion. In addition to the beat noise, other noise sources such as dark current, shot noise and thermal noise also affect the electrical signal. ${ }^{39}$ After conversion, the signals arising from microdiversity are recombined and filtered (electr. filter block in Figure $3 \mathrm{~A}$ ) to minimize the noise power before the electrical preamplification.

\subsubsection{Satellite: RF chain}

The satellite RF processing is undertaken on the DVB-S2x analog waveform and follows either the optical-electrical conversion in case of ATS or the DAC in digital transparent scheme (DTS). It first involves a frequency up-conversion to bring the analog signal to the desired working frequency of the IMUX filters. These filters synthesize the aggregate signal into separate subchannels that are then amplified using an HPA and fed to the OMUX filter to be separated into the desired downlink beams. Before the amplification, an ideal AGC is assumed to bring the signal to the desired power level. We further assume that the HPA is driven in its linear region of operation to focus the study on the impact of optical transmissions.

\subsection{5 $\quad$ User terminal}

The UT processing chain is composed of a receive square-root raised cosine (SRRC) filter, a least mean squares (LMS) linear equalizer to counteract the memory effects induced by the satellite filters, and demodulation and decoding processes of the DVB-S2x standard.

\section{3 | OPTICAL CHANNEL}

The optical uplink channel model for GEO scenario comprises (a) received power time series generator useful for detailed error performance assessment of physical layer transmission techniques and (b) CFLOS time series generator useful for OGSN sites selection and dimensioning.

\section{$3.1 \quad$ Received power time series}

In this subsection, the methodology for the computation of received power time series for the uplink using collimated Gaussian beam waves ${ }^{20}$ will be presented. In the reported methodology, we consider only weak turbulence, whereas the spatial spectrum of refractive index is modeled according to Kolmogorov spectrum. ${ }^{20}$ The structure constant is modeled according to the modified $\mathrm{H}-\mathrm{V}$ model ${ }^{18}$ so as the altitude of the ground stations, day/night conditions and the root mean squared (RMS) wind speed are taken into account, 


$$
\begin{aligned}
C_{n}^{2}(h) & =A_{0} \exp \left(-H_{G S} / 700\right) \exp \left(-\left(h-H_{G S}\right) / 100\right) \\
& +5.94 \cdot 10^{-53} \cdot\left(\frac{u}{27}\right)^{2} h^{10} \exp (-h / 1000) \\
& +2.7 \cdot 10^{-16} \exp (-h / 1500)
\end{aligned}
$$

where $H_{G S}(m)$ is the altitude of ground station, $u$ is the RMS wind speed, and $A_{0}\left(m^{-2 / 3}\right)$ is the refractive index structure parameter at ground level depending on the day and night conditions. The RMS wind speed is computed according to Bufton model. ${ }^{20}$ The value of $A_{0}$ is defined in Table 1. A second important metric for the characterization of turbulence strength is the Fried parameter, $r_{0}$, which is defined through:

$$
r_{0}=\left\{0.42 \sec (\zeta) k^{2} \int_{H_{G S}}^{H} C_{n}^{2}(h) d h\right\}^{-3 / 5},
$$

where $\zeta$ is the zenith angle, $k=2 \pi / \lambda$ with $\lambda$ being the wavelength used and $H$ is the satellite altitude. Furthermore, on-board the satellite, we consider a point detector armed with Gaussian lens placed some meters $(2 \mathrm{~m})$ in front of the detector for counteracting the effects of beam spreading in a nonturbulent medium. ${ }^{20}$

In this step, the main expression for received power time series is presented below so as all the main components are sufficiently described.

$$
P_{R}=P_{T} \cdot G_{T} \cdot G_{R} \cdot \eta_{T} \cdot \eta_{R} \cdot L_{a t m} \cdot F S L \cdot I_{\text {tot }},
$$

where $P_{R}$ and $P_{T}$ are the received and transmitted powers respectively, both in Watts. $G_{T}, G_{R}$ are the antenna gains and $\eta_{T}$ and $\eta_{R}$ are their efficiencies at the transmitter and receiver, respectively. $L_{a t m}$ is the atmospheric loss excluding the turbulence effects, FSL is the free space losses and $I_{\text {tot }}$ is the instantaneous irradiance considering both the turbulence effects (scintillation and beam wander) and the beam spreading due to diffraction. Atmospheric losses consist of the atmospheric transmittance computed using the SpectralCalc ${ }^{50}$ software tool, the cirrus transmittance using the expression (3.5.1) from Degnan ${ }^{22}$ and the losses occurred due to Mie and Rayleigh scattering computed according to ITU-R P. $1622 .{ }^{51}$ These parameters depend on the altitude of the station and the elevation angle of the link, resulting in higher losses for low altitudes and low elevation angles. In this contribution, we separate the turbulence effects into scintillation effects and beam wander and spreading effects. ${ }^{21}$ The instantaneous irradiance due to scintillation is generated through the time series of log-amplitude using SDEs driven by fractional Brownian motion. ${ }^{52}$ The variance of log-amplitude is calculated for fully tracked beams. ${ }^{19}$ Beam wander is considered Rayleigh distributed. The beam spreading effects are calculated using the Strehl ratio. ${ }^{19}$ Finally, as presented in Andrews, ${ }^{19}$ the maximum Strehl ratio (less turbulence) is achieved until $W_{0}=0.1 r_{0}$. Thus, in our analysis, we compute the effective radius $W_{0}(m)$ of Gaussian beam in accordance with the calculated Fried parameter.

\section{2 | Optimum selection of OGSN sites}

In this section, the methodology for the selection of OGSN sites in order to achieve specific CFLOS probabilities for GEO scenario is reported. The objective of this analysis is to present some methods to select the minimum number of OGS that are required in order to guarantee a specific availability percentage. In our analysis, we consider a $99.9 \%$ availability percentage. This procedure is based on the analytical methodology presented in Lyras et al, ${ }^{13}$ where a stochastic dynamic model for the generation of integrated liquid water content (ILWC) time series is reported. In this methodology, SDEs are employed, whereas the temporal and spatial variability of clouds and their microphysical properties are taken into account. This methodology takes into consideration both the elevation angle and the altitude of OGS for the computation of CFLOS probabilities among others. This approach is based on the assumption that ILWC can be sufficiently described by log-normal distribution, whereas the statistical parameters which are used for the synthesis of ILWC time series are derived from ITU-R P.840. ${ }^{53}$ In this work, an ON/OFF channel with the presence of clouds is considered as in Perlot and Perdigues-Armengol ${ }^{15}$ and Sanchez et al. ${ }^{16}$ A snapshot of the cloud coverage time series at Nemea Greece, assuming a GEO satellite and $46^{\circ}$ elevation angle, is presented in Figure 4 . The main steps of this procedure are briefly reported here, and they have been analytically presented in Lyras et al. ${ }^{13}$ :

1. Time series of ILWC fields correlated both on temporal and on spatial domain are synthesized using SDEs.

TAB LE 1 Value of the refractive index structure parameter

\begin{tabular}{lr} 
Condition & $A_{0}\left(\mathrm{~m}^{-2 / 3}\right)$ \\
Day & $1.7 \cdot 10^{-13}$ \\
Night & $1.7 \cdot 10^{-14}$ \\
\hline
\end{tabular}



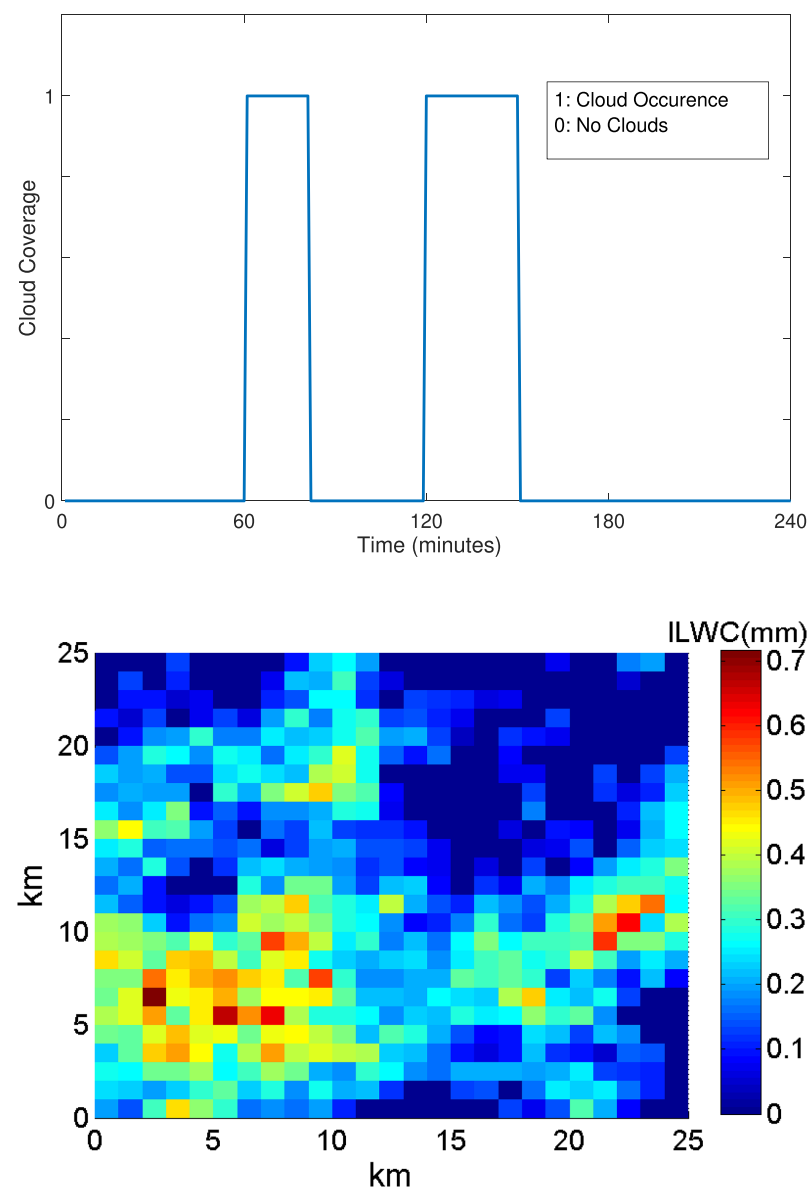

FIG URE 4 Cloud coverage time series for ON/OFF channel [Colour figure can be viewed at wileyonlinelibrary.com]
FIGURE 5 Integrated liquid water content (ILWC) two-dimensional field for a specific time instance [Colour figure can be viewed at wileyonlinelibrary.com]

2. Time series of clouds vertical extent is computed.

3. Cloud coverage time series are computed taking into account the whole slant path, the elevation angle, and the altitude of the ground stations.

4. CFLOS probability is computed from cloud coverage time series.

An ILWC field for a specific time instance of Step 1 of the proposed algorithm is shown in Figure 5.

\subsection{1 | OGSN sites selection considering CFLOS optimization}

This methodology is based on the joint CFLOS of the provided stations with an objective of targeting a given availability percentage (e.g., 99.9\%) using the minimum number of stations and considering only CFLOS. The following steps are taken in this methodology:

1. Time series of cloud coverage for each station are computed; these time series are spatially and temporally correlated between the different locations of the stations.

2. The stations are sorted in descending order of CFLOS probability.

3. Starting from the station with the highest CFLOS probability and going down on the ordered list of Step 2, new stations are added to the pool of selected stations until a target percentage (e.g., 99.9\%) of availability is achieved. The system availability is computed as the probability of having at least one station in the pool not affected by clouds.

\subsubsection{OGSN sites selection considering CFLOS and received power (turbulence effects) optimization}

This methodology tries to optimally select the ground stations so as mainly the effects of turbulence are minimized, and the received power is increased. When the link is not affected by clouds, turbulence represents the dominant fading mechanism which deteriorates the performance of the system. To this end, for the selection of the stations not only the CFLOS but also the Fried parameter will be considered. The latter provides 
information about the strength of turbulence and as a result about the received power. The strength of turbulence depends on the night/day, the wind speed, the altitude of the ground station, and the elevation angle among others. Low values of the altitude above the earth's surface and low elevation angles result in higher turbulence effects. This is because the atmosphere is denser near the earth's surface and lower elevation angles translate to longer path lengths. These parameters, together with the refractive index structure constant, are incorporated in the expression for the calculation of the Fried parameter (2). For this methodology, the ensuing steps will be followed:

1. Time series of cloud coverage for each station are computed; these are spatially and temporally correlated.

2. Fried parameter for each station is estimated taking into account the elevation angle and the altitude of the station.

3. The stations with CFLOS lower than a threshold are not considered in the optimization; a value of $65 \%$ is chosen in this paper.

4. Order the remaining stations with CFLOS more than $65 \%$ in descending order of Fried parameter.

5. Starting from the station with the highest value of Fried parameter and going down on the ordered list of Step 4, new stations are added to the pool of selected stations until a target percentage (e.g., 99.9\%) of availability is achieved. The system availability is computed as the probability of having at least one station in the pool not affected by clouds.

\section{4 | DIVERSITY TECHNIQUES FOR FSO COMMUNICATIONS}

In this section, we detail the microdiversity and macrodiversity techniques used to mitigate the variations in received power due to turbulence in CFLOS conditions and those due to clouds, fog, and rain respectively.

\section{1 | Microdiversity}

Microdiversity aims to mitigate the turbulence related impairments by exploiting multiple transmit lasers and/or optical receptors. Several diversity techniques have been reported in literature on digital communications ${ }^{54}$ including maximal ratio combining (MRC), equal gain combining (EGC), maximal ratio transmission (MRT), ${ }^{55}$ space time block codes (STBC), ${ }^{56}$ eigen-beamforming, ${ }^{57}$ selection combining, and RC. ${ }^{29}$ However, an application of these techniques to the current scenario is not straight forward because of the following:

- A single receiver is considered towards reducing the payload complexity in view of technology evolution pace.

- Acquiring phase of the optical channel on-board is not feasible for the given set-up leading to noncoherent processing at the on-board receiver.

In view of these, many of the aforementioned combining techniques are not applicable, leading to the use of modified RC described below.

\subsection{1 | Modified RC}

In the traditional RC where the streams combine over the air, a power gain can be achieved when under streams add up coherently. However, as shown in Mengali et al., ${ }^{38}$ the traditional RC scheme for optical feeder links performs poorly due to the possibility of destructive interference among the spatially separated streams. Hence, a viable diversity technique for the considered scenario, involves RC, but using additional resources. The same waveform could be transmitted from different transmitter on separate wavelength or polarization. ${ }^{26}$ The streams received on different polarizations/frequencies from the spatially separated CFLOS are processed by different receiver chains. A noncoherent detection is performed and the resulting signals are then combined after the photodiode. While the spatial separation provides for independent channel gains, the use of wavelength/ polarization ensures that the streams can be processed separately at the receiver rather than of their superposition.

\section{2 | Macrodiversity}

Macrodiversity allows the possibility of exploiting spatial diversity by transmissions through multiple CFLOS. In the case of $N+P$ macrodiversity, $N$ active CFLOS are used with the possibility to switch to $P$ idle OGS, based on the channel conditions. Thus, the $N$ CFLOS provide multiplexing, whereas the P CFLOS provide diversity. The treatment in this section builds on the OGSN selection of Section 3.2. In particular, given a selected list of CFLOS, the current investigation tries to find out the system performance assuming the selection of the the best $n(\leq N)$ OGS for each cloud realization. The possibility of having $n<N$ arises from the fact that clouds may block more than $P$ stations. In our work, we assumed that the total 
system capacity is equally distributed among the $N$ active gateways. This leads to the practical situation that a portion of the system capacity is lost when the number of available CFLOS is less than $N$. On the other hand, this also implies a lower cost per OGS and the possibility of fully exploiting the two types of progressive deployment. The first one, progressive w.r.t system capacity, is the one in which full capacity is achieved over time by successive introduction of smaller active CFLOS. This enables the network to become operational before its full deployment and allows the operator to easily adjust to higher throughput demands by increasing the number of active gateways. The second kind, progressive w.r.t. system availability, is the one in which a gradual deployment of the redundant CFLOS is performed to reduce the network outage probability and improve quality of service (QoS).

A special case involves $N=1$ whence only one active OGS is used to uplink all the feeder link traffic. This minimizes the number of OGS and reduces the portion of traffic lost due to cloud blockage. Doing so, on the other hand, imposes additional complexity requirements at OGS and satellite side due to bandwidth used and inhibit progressive deployment of capacity without switching to a $N>1$ paradigm.

It should be noted that macrodiversity can be combined with microdiversity with each OGS employing the modified RC discussed in Section 4.1. Assuming a perfect handover between affected OGS and an idle OGS and absence of correlation between the CFLOS with regards to blockage, we now proceed to evaluate the performance of a $N+P$ system analytically. While the first assumption can be realized with prediction of blockage and synchronization, larger geographic separation between the CFLOS corroborates the independent blockage assumption. In particular, employing the performance of individual OGS, we obtain the throughput and outage for the system.

\subsection{1 | Throughput and outage assessment}

Let $F_{s}(\gamma)$ denote the FER of OGS $s \in\{1,2, \ldots, N+P\}$ for a downlink SNR $\gamma$; the normalized throughput of this OGS is then, $T_{s}(\gamma)=1-F_{s}(\gamma)$. Further, we assume any OGS $s$ can be in either of the two states:

1. State 1: CFLOS state, with probability $p_{s}$ obtained from the channel model (kindly refer to Section 3).

2. State 0: Blockage, with probability $1-p_{s}$.

It is clear that in a OGSN, a number of $2^{(N+P)}$ distinct cloud formations con arise over its $N+P$ CFLOS. Let $f_{i}, i \in\left\{1,2, \ldots, 2^{N+P}\right\}$ be the $1 \times(N+P)$ binary row vector depicting the CFLOS states for the ith possible cloud formation; in particular, the Ith element of $f_{i}$, denoted by $f_{i}(I)$, depicts the state of OGS I when the ith cloud formation occurs. Let now $\Theta$ represent the $2^{(N+P)} \times(N+P)$ state probability matrix, describing the probabilities for all the possible system states. The element in row $r$ and column $t, \boldsymbol{\Theta}_{r, t}$, will be equal to $p_{s}$ if OGS $s$ experiences CFLOS in cloud formation identified by $r$ and $1-p_{s}$ otherwise, that is, $\boldsymbol{\Theta}_{r, t}=\operatorname{Prob}\left\{\underline{f}_{r}(t)\right\}$ where Prob $\{\cdot\}$ denotes probability operation.

Example: Consider a system with a total of three stations $(N+P=3)$ and consider the cloud formation event where OGS 1 experiences CFLOS while OGS 2 and 3 are covered by clouds. Denoting this OGSN state as $i$, we have $\underline{f}_{i}=[1,0,0]$. Further, the ith row of $\boldsymbol{\Theta}$ takes the form $\left[p_{1}, 1-p_{2}, 1-p_{3}\right]$. Assuming independent cloud realizations at the separate CFLOS, the probability of the event $\underline{f}_{i}$ happening is simply found by multiplying the elements of the ith row of $\Theta$, that is, $\operatorname{Prob}\left\{\underline{f}_{i}\right\}=p_{1} \cdot\left(1-p_{2}\right) \cdot\left(1-p_{3}\right)$.

The system performance, when macrodiversity is employed, is then found according to the following steps:

1. Set $n=N$.

2. Find the set $\mathcal{C}_{n}$ of all the possible combinations of groups of $n$ out of $N+P$ OGS. The cardinality of $\mathcal{C}_{n}$ is $\frac{(N+P) !}{n !(N+P-n) !}$.

3. For each combination $c \in \mathcal{C}_{n}$, define $\mathcal{S}_{c}$ as the set of CFLOS selected in $c$ and $T^{c}(\gamma)$ as the normalized throughput of $c$ for a SNR $\gamma$ :

$$
T^{c}(\gamma)=\frac{1}{N} \sum_{s \in \mathcal{S}_{c}} T_{s}(\gamma)
$$

4. Order each OGS combination in descending order of throughput, that is, we let $\mathcal{C}_{n}=\left\{c_{1}, c_{2}, \ldots, \mathcal{C}_{\left|\mathcal{C}_{n}\right|}\right\}$ with $T^{c_{1}}(\gamma) \geq T^{c_{2}}(\gamma) \ldots T^{c_{1} \mid}$ where $\left|\mathcal{C}_{n}\right|$ refers to the cardinality of $\mathcal{C}_{n}$.

5. Set $k=1$

(a) Choose $c_{k}$ and find $\mathcal{S}_{c_{k}}$ to be the set of stations active in $c_{k}$;

(b) Find the set $r_{c}$ of all the rows in matrix $\Theta$ for which all the stations in $\mathcal{S}_{c}$ experience CFLOS;

(c) Compute the probability $\rho_{c_{k}}$ of combination of stations $c$ to be selected as the sum of the probabilities of all events identified by rows $r_{c}$ and delete those rows from $\Theta$; 
(d) Set $k=k+1$ and repeat Step (a) until $k=\left|\mathcal{C}_{n}\right|$.

6. Set $n=n-1$ and repeat steps $2-5$ until $n=0$ whence $\Theta$ will be reduced to a single row representing the event of clouds covering all the $N+P$ CFLOS.

The normalized system throughput is calculated as

$$
T_{\mathrm{SYS}}(\gamma)=\sum_{n=1}^{N} \sum_{c \in \mathcal{C}_{n}} T^{c}(\gamma) \cdot \rho_{c}
$$

and system average FER as

$$
\operatorname{FER} R_{\mathrm{SYS}}(\gamma)=1-\mathrm{T}_{\mathrm{SYS}}(\gamma)
$$

\section{5 | PERFORMANCE ANALYSIS OF THE OGSN}

In this section, we consider both the system level and link level performance assessments. The key elements for such assessments including the OGS selection based on channel models, SNR calculations incorporating various noise contributions, and the choice of the parameters are presented first, followed by detailed simulation results. Note that the system performance results were evaluated within the ONSET project by SES from the commercial telecom satellite operator's perspective. ${ }^{40}$

\subsection{OGSN dimensioning}

In this subsection, the results of OGSN dimensioning analysis using the two approaches of Section 3.2 are presented. The main targets of this scenario as defined before are (a) pan European coverage which is ensured using the ASTRA satellite at $19.2^{\circ}$ East, (b) $99.9 \%$ availability using macrodiversity technique with the minimum number of OGS, and (c) reduction of the potential total cost by using already existing infrastructure and gateway locations (incl. those that SES has access to) and some additional astronomical sites in high altitudes to ensure the high availability. Figure 6 illustrates the input pool of OGSN sites, all having an elevation angle in excess of $30^{\circ}$, considered in the optimization analysis.

FIGURE 6 Map of input pool of optical ground station network (OGSN) sites considered in the optimization analysis [Colour figure can be viewed at wileyonlinelibrary.com]

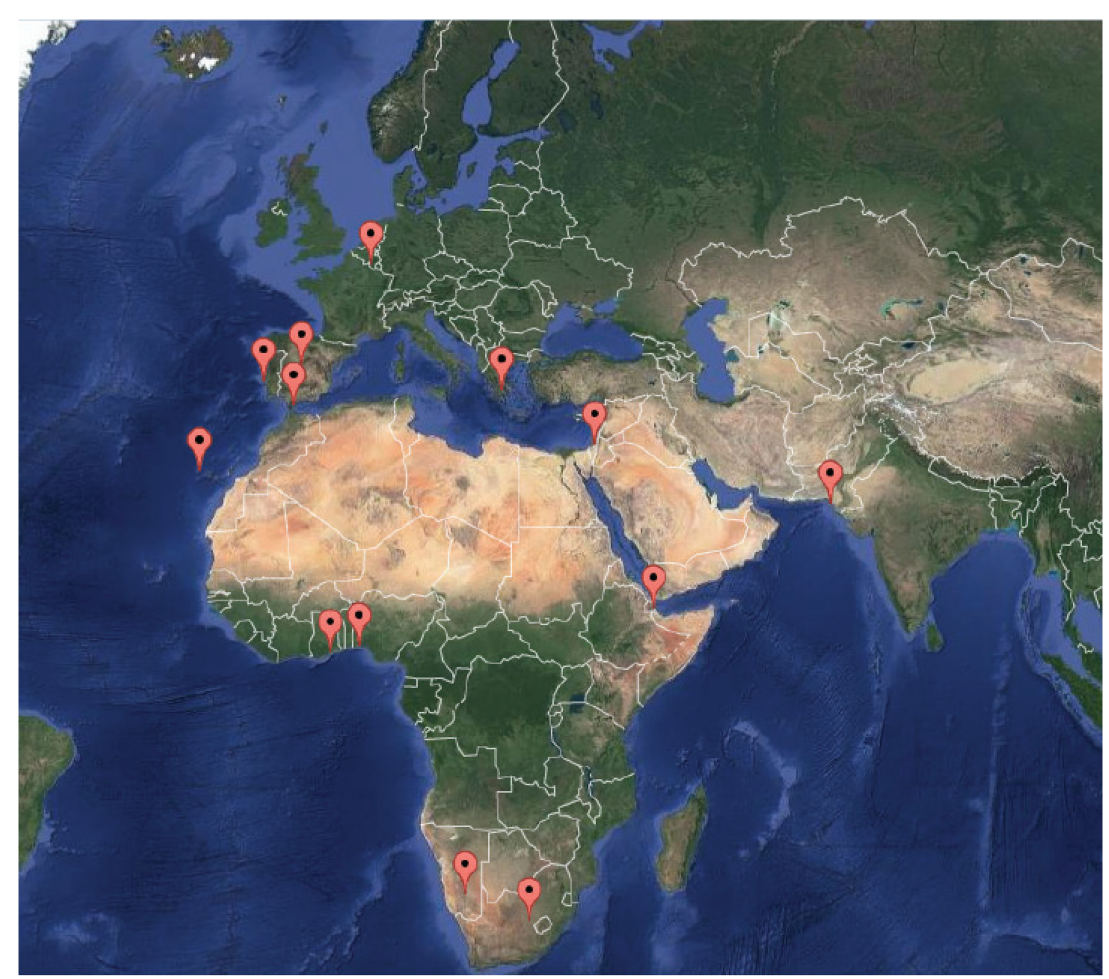


Firstly, the minimum number of the stations so as $99.9 \%$ availability is achieved will be defined according to the methodology presented in Section 3.2.1. Table 2 provides the selected OGSN sites considering the CFLOS optimization methodology. In the last column, the system availability is shown, that is, in the first row is the probability of the availability of the first station, in the second row, the probability at least one of the two selected is available etc. These four stations ensure more than $99.9 \%$ availability.

Table 3, instead, provides the selected OGSN sites considering the CFLOS and received power methodology illustrated in Section 3.2.2. The selected stations in this case are the ones with the lowest turbulence effects and as a result with the highest Fried parameter. With this methodology, Karachi is not selected. Karachi is favorable as far as the CFLOS is concerned but because of the low elevation angle and the low altitude of the station, the effects of turbulence are severe.

\section{$5.2 \quad$ Received power time series}

We now present the results of the received power time series generation exercise based on the methodology presented in Section 3.1. For these numerical results, the transmitted power per laser is assumed to be $50 \mathrm{~W}$, the transmitter and receiver both have an aperture of $25 \mathrm{~cm}$ and an efficiency of 0.7 , the cirrus cloud thickness for the estimation of cirrus transmittance is assumed to be $2 \mathrm{~km}$, and the wavelength is $1550 \mathrm{~nm}$. Numerical results considering day conditions for the optical links in Madrid and in La Palma are shown in Figures 7 and 8, respectively.

\section{3 | OSNR ratio calculations}

Several noise contributions from the optical processing impact the SNR at the input of the RF transponder and are summarized in this section. The formulations for computing the various noise components presented here are adapted from lizuka. ${ }^{39}$ Given a useful incident signal optical

TAB LE 2 Selected optical ground station network (OGSN) sites considering the cloud-free line of sight (CFLOS) optimization methodology

\begin{tabular}{|llllll}
\hline Location & Lat. $\left({ }^{\circ}\right)$ & Long. $\left({ }^{\circ}\right)$ & Alt. $(\mathrm{m})$ & Elev. angle $\left({ }^{\circ}\right)$ & Sys. availability $(\%)$ \\
\hline La Palma & 28.76 & -17.9 & 2396 & 37.46 & 89.96 \\
\hline Karachi & 24.88 & 66.83 & 18 & 30.1732 & 98.52 \\
\hline Jerusalem & 31.81 & 35.09 & 690 & 49.1422 & 99.73 \\
\hline South Africa & -29.04 & 26.40 & 1380 & 55.199 & 99.94 \\
\hline
\end{tabular}

TAB LE 3 Selected optical ground station network (OGSN) sites considering the cloud-free line of sight (CFLOS) and received power optimization methodology

\begin{tabular}{|llllllll}
\hline Location & Lat. $\left({ }^{\circ}\right)$ & Long. $\left({ }^{\circ}\right)$ & Alt. $(\mathrm{m})$ & Elev. angle $\left({ }^{\circ}\right)$ & $r_{0}(\mathrm{~m})$ & 0.2926 & Sys. availability $(\%)$ \\
\hline La Palma & 28.76 & -17.9 & 2396 & 37.46 & 59.707 & 0.183 & 97.5 \\
\hline Namibia & -25.88 & 17.78 & 1510 & 55.199 & 0.158 & 99.45 \\
\hline South Africa & -29.04 & 26.40 & 1380 & 37.39 & 0.086 & 99.83 \\
\hline Madrid & 40.43 & -4.25 & 864 & 49.1422 & 0.085 & 99.96 \\
\hline Jerusalem & 31.81 & 35.09 & 690 & & \\
\hline
\end{tabular}

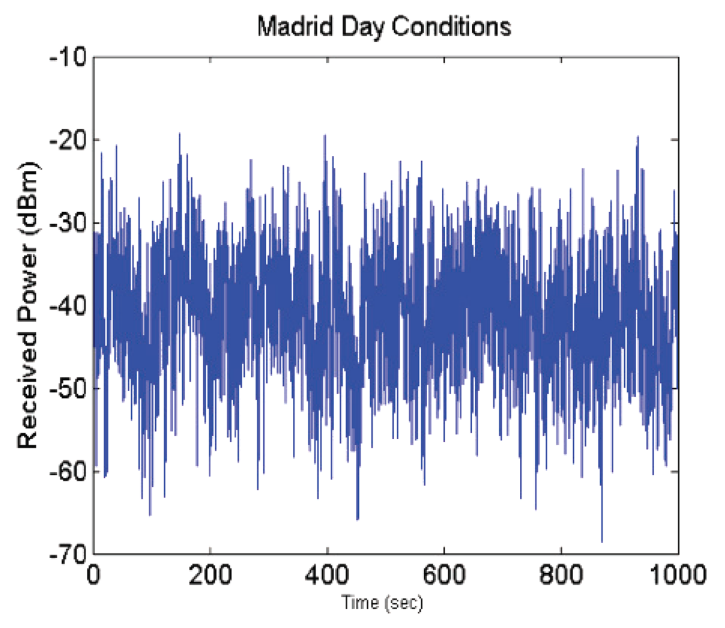

FIGURE 7 Received power time series for Madrid-day conditions [Colour figure can be viewed at wileyonlinelibrary.com] 
FIG URE 8 Received power time series for La Palma-day conditions [Colour figure can be viewed at wileyonlinelibrary.com]

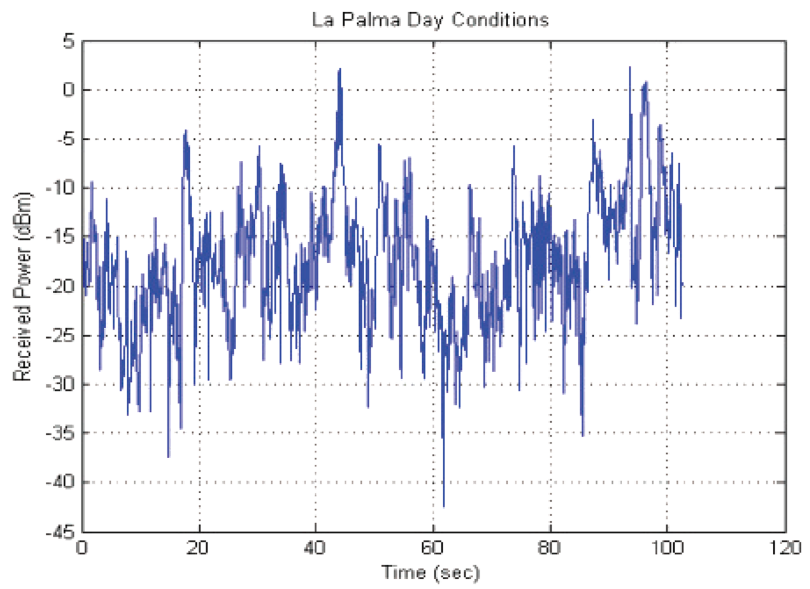

power of $P_{S}$, an EDFA optical preamplifier with optical gain $G$, the electrical current corresponding to the signal at the output of the PIN photodiode can be expressed as

$$
I_{\mathrm{S}}=R \cdot G \cdot P_{\mathrm{S}}
$$

with $R \leq 1$ being the responsivity of the PIN diode.

Background noise: Similar to (7), the current at the output of the photodiode due to background noise can be found as

$$
\begin{gathered}
I_{\text {back }}=R \cdot G \cdot P_{\text {back }}, \\
P_{\text {back }}=m_{t} \cdot \rho_{\text {back }} \cdot B_{o},
\end{gathered}
$$

where $P_{\text {back }}$ is the total background noise power arriving at the PIN receptor. The computation of $P_{\text {back }}$ in (9) follows, ${ }^{58}$ with $m_{t}$ representing the number of optical modes in the system, $B_{\mathrm{o}}$ is the bandwidth of the optical filter, and $\rho_{\text {back }}$ is the power spectral density per-mode of the background radiation. In our study, considering the nature of our link wherein the narrowfield on-board antenna is pointing at earth surface, we consider the combined effect of earth reflection and black-body radiation to compute $\rho_{\text {back. }}{ }^{58}$

ASE noise: The equivalent ASE noise power of the EDFA before amplification, denoted by $P_{\text {ASE, }}$ can be computed as

$$
P_{\mathrm{ASE}}=m_{t} \cdot \rho_{\mathrm{ASE}} \cdot B_{\mathrm{o}}
$$

where $\rho_{\text {ASE }}$ is the power spectral density per-mode of the spontaneous emission. We would like to point out that in our simulations, we used $m_{t}=1$, deriving from the use of a single-mode fiber and the assumption of utilizing a polarization filter prior to the optical preamplification. Then, the ASE noise DC component can be formulated as

$$
I_{\mathrm{ASE}}=R \cdot G \cdot P_{\mathrm{ASE}}
$$

Shot noise: This is present in all currents generated by the photo-detection process, including the dark current and background noise. Considering a generic current $I$ and denoting with $q$ the electron charge, the shot noise power relative to this current can be expressed as

$$
i_{\mathrm{sh}}^{2}=2 q \mid B_{\mathrm{e}}
$$

where $B_{\mathrm{e}}$ is the bandwidth of the electrical filter after the PIN.

Beat noise: On the other hand, the beat noise power can be expressed as follows:

$$
\begin{gathered}
i_{\text {sig-sp }}^{2}=4 I_{S} \cdot I_{\text {ASE }} \cdot \frac{B_{e}}{B_{0}}, \\
i_{\text {sp-sp }}^{2}=I_{\text {ASE }}^{2}\left(2-\frac{B_{e}}{B_{0}}\right) \frac{B_{e}}{B_{0}},
\end{gathered}
$$


where (13) represents the term relative to the product between the useful signal and the ASE noise and (14) represents the term relative to the product of the ASE noise with itself.

RIN: This is signal-dependent noise generated directly at the transmitting laser. This noise is caused by the intensity fluctuations which always happen in the output of a laser source even when a constant injection current is used to power it. This distortion must be taken into account in the total noise computation and takes the following form, ${ }^{59}$

$$
i_{\mathrm{RIN}}^{2}=\rho_{\mathrm{RIN}} \cdot l_{\mathrm{S}}^{2} \cdot B_{\mathrm{e}}
$$

where $\rho_{\text {RIN }}$ represents the power spectral density (normalized w.r.t. to the signal power) of the RIN process.

Thermal noise: In optical systems is usually defined by means of the electrical noise current spectral density $i_{n}$ as

$$
i_{\text {th }}^{2}=i_{\mathrm{n}}^{2} \cdot B_{\mathrm{e}}
$$

We define optical signal-to-noise ratio (OSNR) as the ratio between the useful signal power and the total noise power introduced by the opto-electrical chain. Using previous equations, the OSNR in the DTS can be expressed as

$$
\operatorname{OSNR} R_{d}=\frac{l_{S}^{2}}{i_{\text {beat }}^{2}+i_{\text {RIN }}^{2}+i_{\text {shot }}^{2}+i_{\text {th }}^{2}},
$$

where $i_{\text {beat }}^{2}=i_{\text {sig-sp }}^{2}+i_{\text {sp-sp }}^{2}$ represents the total noise due to beats of the ASE and $i_{\text {shot }}^{2}=2 q\left(I_{S}+I_{\text {ASE }}+I_{d}+I_{\text {back }}\right) B_{\text {e }}$ represents the total shot noise in the chain.

In the analog case, the noise power will remain the same, but the useful signal power is lower due to the power bias introduced at the transmitter.

Let $P_{X}$ denotes the power of the useful transmitted signal, $I_{\text {bias }}$ denotes the amplitude of the bias and $P_{T}$ the total transmitted power including both the useful signal and the bias. One can easily compute the useful power ratio $\beta$ as

$$
\beta=\frac{P_{x}}{P_{T}}=\frac{P_{x}}{P_{X}+l_{\text {bias }}^{2}} .
$$

In our simulations, we choose $I_{\text {bias }}$ as the maximum value of the signal at the output of the transmitter baseband filter. For QPSK modulation, this leads to a value of $\beta=0.222$. The OSNR in the ATS is then

$$
\operatorname{OSNR}_{a}=\frac{\beta l_{\mathrm{S}}^{2}}{i_{\text {beat }}^{2}+i_{\text {RIN }}^{2}+i_{\text {shot }}^{2}+i_{\text {th }}^{2}},
$$

which is slightly different from the same relation found in Mengali et al. ${ }^{38}$ This is due to the fact that ${ }^{38}$ assumed the input/output characteristic of the transmitter laser to relate input electrical amplitude to output light amplitude. In this work, instead, we have adopted the exact relationship where to an input electrical amplitude corresponds an output power (density). ${ }^{39}$

\subsection{Simulation parameters}

FER is considered as the figure of merit with FER less than $10^{-4}$ generally accepted by the community as quasi-error free condition on the userlink. This criteria applies to the current study as the RF link is not changed. These FER results help in determining the system configuration for meeting the requirements.

In all simulations, we assumed a transmit power of $50 \mathrm{~W}$ per laser source and the MODCOD described by QPSK constellation with the Low Density Parity Check (LDPC) code of rate 3/4. Regarding the satellite received power, we assumed the power to remain constant within a single DVB-S2x frame and to change from frame to frame according to the time series generated through the methodology explained in Section 3.1 . This simplifying assumption has been made because the coherence time of the uplink channel, depending on the cut-off frequency of scintillation, ${ }^{60}$ is usually in the order of milliseconds which is much longer than a typical DVB-S2x frame rate in broadband applications. The ADC and DAC have $Q=6$ quantization bits per sample per dimension leading to a total of 12 bits per RF sample. We use the IMUX filter, OMUX filter and the nonlinear HPA specified in the DVB-S2 standard. ${ }^{61}$ We further assume the combined effect of the electrical pre-amplifier and the AGC to result in a power level at the output of the IMUX which is $2 \mathrm{~dB}$ lower than the amplifier saturation power (IBO $=2 \mathrm{~dB}$ ). The other parameters used 
in the simulations are given in Table 4. We assume the satellite to operate in a GEO orbit (ASTRA Satellite at $19.2^{\circ} \mathrm{E}$ is in fact considered). Regarding separation in frequency for the modified RC, the guidelines for the DWDM grid specified in previous study ${ }^{62}$ were assumed in this work which indicate a frequency separation between channels of multiples of $12.5 \mathrm{GHz}$ (roughly $0.1 \mathrm{~nm}$ ). This being said, in the DTS case, the separation is dependent on the required bandwidth expansion. For the case of 12 bits per symbol analyzed here and OOK modulation (meaning an optical/electrical bandwidth of $18 \mathrm{GHz}$ ), the actual spacing needs to be at least $25 \mathrm{GHz}$. At this point, note that similar assumption with respect to the DWDM grid specifications of ${ }^{62}$ was also followed in the EU FP7 project BATS according to the results published in Roy et al. ${ }^{32}$ In that case, the assumed frequency spacing was actually: $50 \mathrm{GHz}$ for digital coded transmission, $100 \mathrm{GHz}$ for digital uncoded transmission, and $25 \mathrm{GHz}$ for analog transmission.

\section{5 | Performance evaluation: ATS and DTS systems}

We assume a quantization of $Q=6$ bits per dimension per sample in the DTS leading to a bandwidth expansion of 12 for the transmission with OOK. Given that the complexity of an optical feeder link system strongly depends on the number of needed optical chains, we decided to compare analog and digital assuming the same useful signal bandwidth (RF waveform) per chain. This implies that without considering the additional chains due to microdiversity, the two systems would have the same number of chains for given target system throughput.

\section{6 | Performance evaluation: Impact of altitude}

Figure 9 shows the performance of the DTS for the CFLOS sites identified in Table 2 assuming CFLOS conditions both at their original altitude Figure 9A and assuming same coordinates but at higher hypothetical altitudes Figure 9B. New hypothetical altitude was set to $1500 \mathrm{~m}$ for stations located below that value (i.e., Jerusalem, Karachi, and South Africa) and kept unchanged for the station already above that value (Palma). This exercise was undertaken towards enhancing the FER performance of the single stations. Results clearly indicate that the low altitude stations are severely impacted by the worse irradiance turbulence. An improvement of around two orders of magnitude is achievable by increasing the altitude of South Africa and Jerusalem. Another interesting conclusion can be drawn regarding the impact of the elevation angle of the stations. In fact, it can be seen that despite the increase in altitude stations at low elevation angle, like Karachi, experience worse performance with respect to stations at same altitude but higher elevation angle, like Bloemfontain in South Africa.

\section{7 | Performance evaluation: microdiversity}

We considered up to four separated transmitters in our microdiversity scheme. Because the spatial coherence length of the satellite optical channel is in the order of centimeters, ${ }^{18}$ the different transmitted beams are assumed to experience an independent realization of the turbulence channel. As mentioned in Section 5.4, we assumed a fixed power per laser of $50 \mathrm{~W}$, leading to an increase in the total transmitted power linearly dependent on the microdiversity order employed.

\subsection{1 | Digital transparent}

To analyze the impact of microdiversity in a DTS, for reasons of space, we focused on the results for a single station (Jerusalem) and changed the order of the transmitter microdiversity. Results are illustrated in Figure 10 together with a lower bound on the achievable FER (RF uplink in the figure). This lower bound was obtained using the similar parameters to simulate a purely RF system characterized by an ideal noiseless uplink channel.

TABLE 4 Parameters used for the simulations

\begin{tabular}{|c|c|c|c|}
\hline Parameter & Value & Parameter & Value \\
\hline$\lambda$ & $1550 \mathrm{~nm}$ & $\rho_{\text {ASE }}$ & $2^{-19} \mathrm{~W} / \mathrm{Hz}$ \\
\hline$B_{e}$ & $1.5 \mathrm{GHz}$ (ATS), $18 \mathrm{GHz}$ (DTS) & $\rho_{\text {back }}$ & $7.6^{-25} \mathrm{~W} / \mathrm{Hz}$ \\
\hline$B_{0}$ & $12.5 \mathrm{GHz} / 18 \mathrm{GHz}$ (DTS) & $\rho_{\mathrm{RIN}}$ & $-160 \mathrm{dBc} / \mathrm{Hz}$ \\
\hline G & $50 \mathrm{~dB}$ & $I_{\mathrm{d}}$ & $10^{-10} \mathrm{~A}$ \\
\hline$R$ & 0.5 & $i_{\mathrm{n}}$ & $10^{-11} \mathrm{~A} / \sqrt{\mathrm{Hz}}$ \\
\hline
\end{tabular}

Abbreviations: ATS, analog transparent scheme; DTS, digital transparent scheme. 


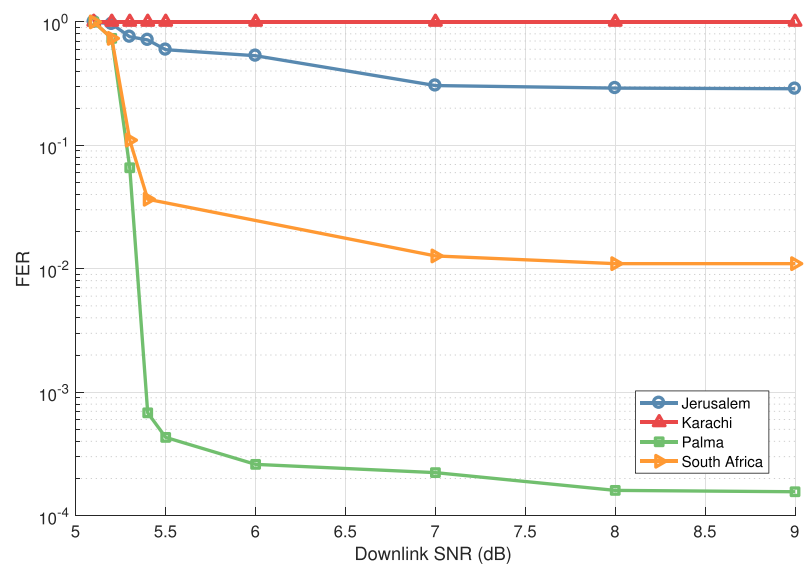

(A) Original Altitudes.

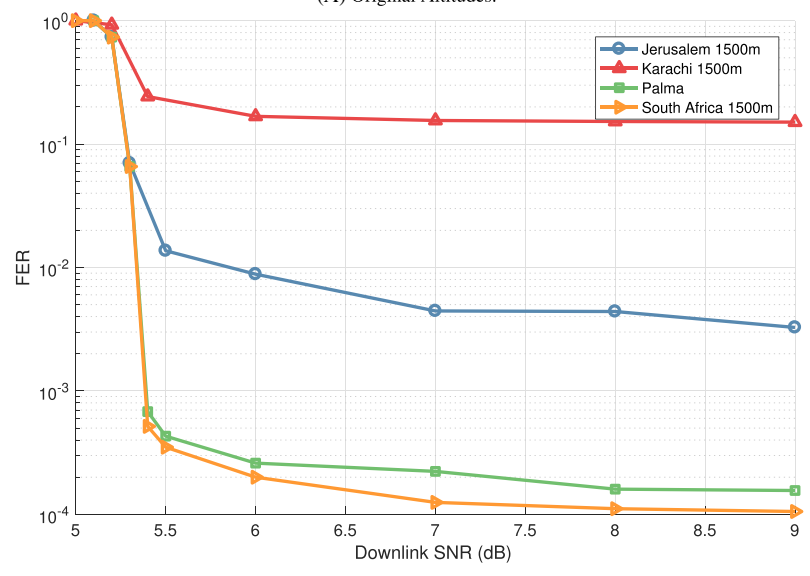

(B) New Hypothetical Altitudes.

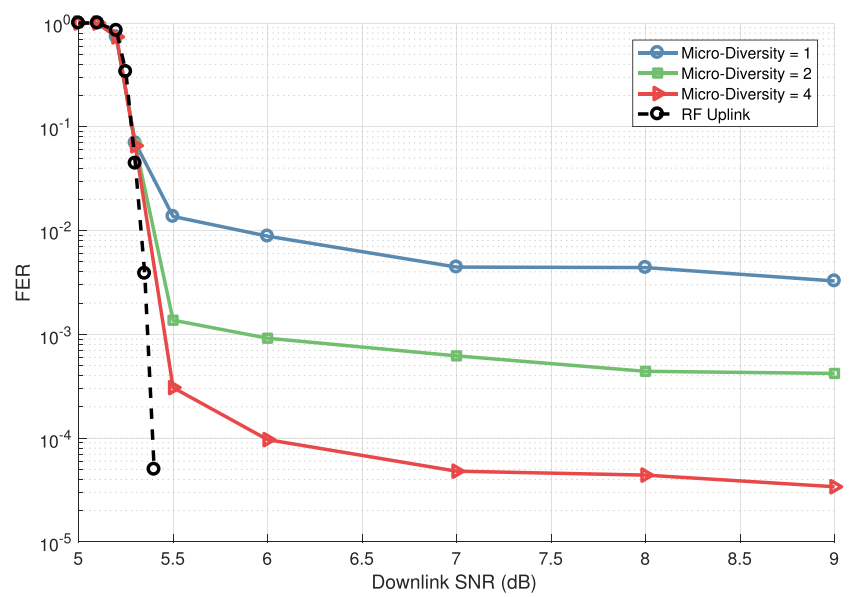

FIGURE 9 Frame error rate (FER) performance in cloud-free line of sight (CFLOS) conditions for different optical ground station (OGS) locations both at their original and new hypothetical altitudes [Colour figure can be viewed at wileyonlinelibrary.com]
FIGURE 10 Microdiversity digital transparent scheme (DTS): Jerusalem at $1500 \mathrm{~m}$ [Colour figure can be viewed at wileyonlinelibrary.com]

\subsection{2 | Analog transparent}

To analyze the impact of microdiversity in a ATS, we also focused on the same stations (Jerusalem) and changed the order of the transmitter microdiversity. Results are illustrated in Figure 11. It is important to remind that, as explained in Section 5.5, we are assuming same useful RF bandwidth per chain to compare analog and digital performances. It can be seen that despite the wasted power in the bias, the ATS is outperforming the DTS with the selected choice of parameters. This is due to the bandwidth expansion in DTS which mandates the use of larger filters resulting in more noise. It is important to recall that for the analog case we assumed an ideal laser characteristic and no stream aggregation. Including a more realistic characteristic and taking into account the stream aggregation would reduce the value of $\beta$ thus lowering the performance of the ATS. 
FIGURE 11 Microdiversity analog transparent scheme (ATS): Jerusalem at $1500 \mathrm{~m}$ [Colour figure can be viewed at wileyonlinelibrary.com]

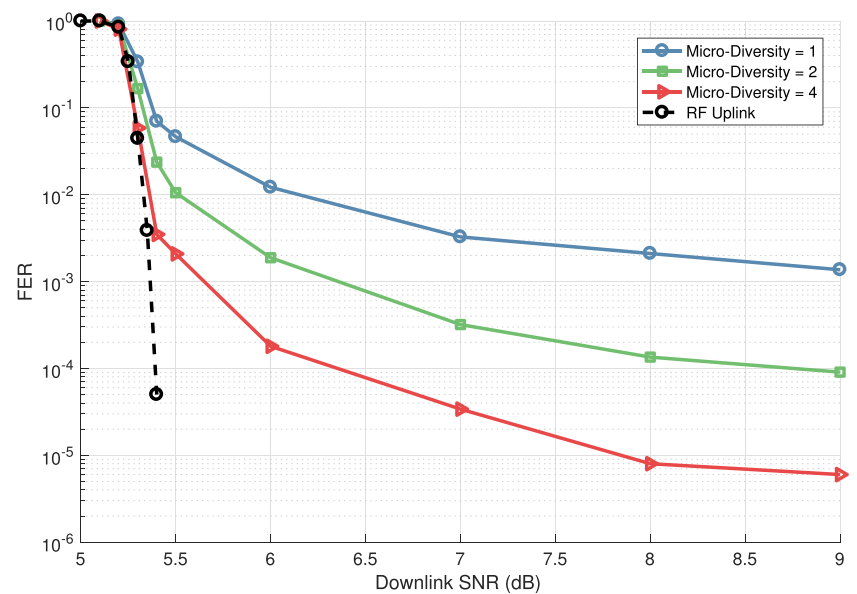

FIGURE 12 Performance of optimal and estimated on-off keying (OOK) threshold selection at Jerusalem location with new hypothetical altitude [Colour figure can be viewed at wileyonlinelibrary.com]

FIGURE 13 Average frame error rate (FER) system performance for digital transparent scheme (DTS) employing microdiversity and different levels of macrodiversity [Colour figure can be viewed at wileyonlinelibrary.com]
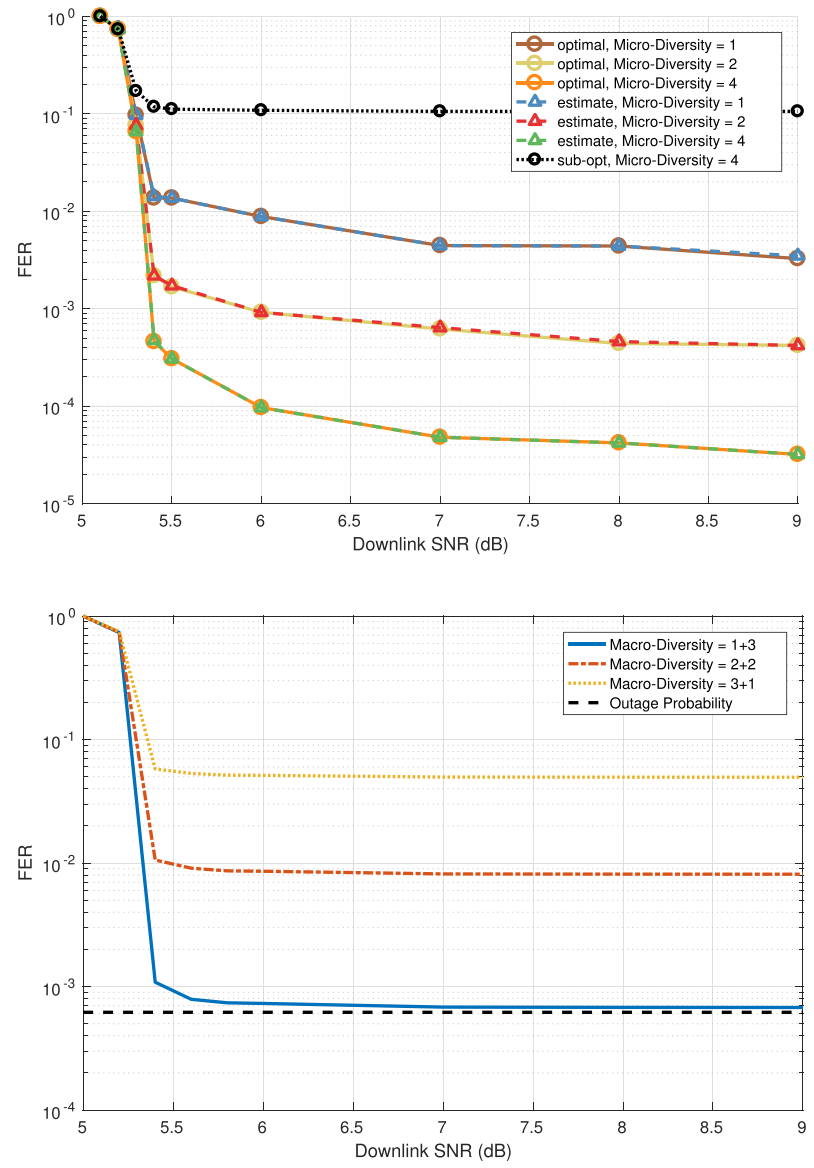

\section{8 | Performance evaluation: OOK decoding threshold}

The optical modulation scheme used in the DTS is OOK. It is known that OOK is particularly sensitive to the choice of the detection threshold, with the optimal choice depending on the instantaneous irradiance. The sensitivity to the threshold is exacerbated by the severity of irradiance scintillation present in a Earth-to-Satellite link. Adaptive threshold selection based on average received irradiance is considered to enhance the detection performance of OOK. A simple algorithm involves using the electrical amplitude averaged over a fixed time window prior to detection as threshold. While this averaging will include the contribution of the noise generated in the opto-electrical conversion process, it yields an estimator of the optimal threshold if the transmitted bits are equiprobable, and the noise process is zero mean. A comparison of the performance using an optimal threshold and an estimate based on the first 1000 bits of the frame is shown in Figure 12 for various levels of microdiversity and assuming the location of Jerusalem with the new hypothetical altitude. The result obtained by employing a microdiversity of order 4 and a suboptimal adaptive threshold scheme in which the threshold is set to the optimal value once every two frames, and kept constant for the following one is shown in dotted black line for reference. This result clearly shows that the performance of OOK is highly sensitive to the correct selection 
of the threshold and a slow update leads to unacceptable results. The other lines, on the other hand, show that despite the noise and the limited averaging window (1000 bits correspond to less than 1/100th of the frame duration in our simulation setup), performance with the estimated threshold closely matches that of the optimal threshold.

\section{9 | Performance evaluation: macrodiversity}

We computed the system-level performance of the macrodiversity scheme as explained in Section 4.2. For the results, we focused on the pool of stations identified in Table 2 resulting in $N+P=4$, and we limited ourselves to the DTS. Figure 13 shows the performance for varying $N$ with the use of microdiversity at each single station. In the figure, the lower bound on FER, identifying the probability of having clouds over all the stations, is depicted with a black dashed line. As discussed in Section 4.2, the performance with $N>1$ is worse due to the higher probability of having a number $n<N$ of CFLOS experiencing CFLOS.

\section{6 | CONCLUSIONS}

The paper represents the outcome of a study activity on satellite systems with optical feeder link and RF user links. For a pan-European coverage scenario, the paper explored various system aspects including channel models and transmission techniques towards devising a feasible system. As system constraints, the functionality was confined to transparent payloads where two architectures (analog transparent and digital transparent) were considered. For these settings, microdiversity and macrodiversity techniques for fade-mitigation were considered. An end-to-end physical layer system simulator capable of detailed forward link performance assessment for various GEO system scenarios has been developed providing interesting trade-offs and insights on the system functionalities as well as opening novel challenges for enhancing the performance. Results obtained using both digital and analog transmission options coupled with transparent payloads clearly highlight the significant impact of the optical uplink on the end-to-end performance. The presented FER results clearly show an error floor often above $10^{-4}$ even when considering microdiversity and a simulated altitude of $1500 \mathrm{~m}$. Such values would not allow a satellite system to keep the desired reliability using only optical feeder links without additional countermeasures such as adaptive optics (AO) precompensation. ${ }^{63}$ The novelties of channel model, rigorousness in incorporation of impairments in the simulator and an extensive simulation campaign indicates the potential of the work to serve as a framework for evaluating performance of evolving techniques.

\section{ACKNOWLEDGMENTS}

The work presented in this paper was carried out under the project ONSET (Optical feeder links study for satellite networks - ESA contract no. 40000113462/15/NL/NDe). ONSET was funded by the Government of Luxembourg (Ministry of Economy) through an ESA contract in the Luxembourg Third Party Programme, for which Prime Contractor was SES TechCom S.A. The views expressed herein can in no way be taken to reflect the official opinion of the European Space Agency (ESA).

The work of Alberto Mengali was carried out during his Ph.D. at University of Luxembourg and supported by the National Research Fund, Luxembourg under AFR grant for PhD project (reference 10064089) on "Reliable Communication Techniques for Future Generation Satellite Systems". He is currently with ESA.

The authors would like to thank the Government of Luxembourg and ESA for their funding support. The authors would also like to thank particularly Dr. Pedro Baptista from ESA and Guy Baister from Thales Alenia Space Switzerland for their valuable support during the ONSET project.

\section{ORCID}

Alberto Mengali (D) https://orcid.org/0000-0001-6413-7151

Bhavani Shankar Mysore Rama Rao (D) https://orcid.org/0000-0002-3638-1349

Farbod Kayhan (D) https://orcid.org/0000-0002-1733-5435

Athanasios D. Panagopoulos (D) https://orcid.org/0000-0003-4716-3328

Konstantinos Liolis (D) https://orcid.org/0000-0003-2337-8148

\section{REFERENCES}

1. Muhammad M, Giambene G, Cola Td. Channel prediction and network coding for smart gateway diversity in terabit satellite networks. In: 2014 IEEE Global Communications Conference; 2014; Austin, TX, USA:3549-3554. https://doi.org/10.1109/GLOCOM.2014.7037358

2. Gharanjik A, Rao BSMR, Arapoglou P-D, Ottersten B. Large scale transmit diversity in Q/V band feeder link with multiple gateways. In: 2013 IEEE 24th International Symposium on Personal Indoor and Mobile Radio Communications (PIMRC); 2013; London, UK:766-770. https://doi.org/10.1109/ PIMRC.2013.6666239

3. Kyrgiazos A, Evans BG, Thompson P. On the gateway diversity for high throughput broadband satellite systems. IEEE Trans Wirel Commun. 2014;13 (10):5411-5426. 
4. Candreva EA, Suffritti R, Dervin M. Increasing the feeder link efficiency in broadband satellite systems. In: 2014 7th Advanced Satellite Multimedia Systems Conference and the 13th Signal Processing for Space Communications Workshop (ASMS/SPSC); 2014; Livorno, Italy:300-305. https://doi. org/10.1109/ASMS-SPSC.2014.6934559

5. 1st ESA W-Band SATCOM Workshop. WorkShop, ESA/ESTEC Noordwijk, The Netherlands, ESA; 2015. http://congrexprojects.com/2015-events/ 15m17/introduction

6. 5g White Paper. White Paper, NGMN Alliance, NGMN; 2015. https://www.ngmn.org/uploads/media/NGMN_5G_White_Paper_V1_0.pdf

7. Cowley W, Giggenbach D, Calvo RM. Optical transmission schemes for GEO feeder links. In: 2014 IEEE International Conference on Communications (ICC); 2014; Sydney, NSW, Australia:4154-4159.

8. Gharanjik A, Liolis K, Shankar MRB, Ottersten B. Spatial multiplexing in optical feeder links for high throughput satellites. In: 2014 IEEE Global Conference on Signal and Information Processing (GlobalSIP); 2014; Atlanta, GA, USA:1112-1116.

9. Dimitrov S, Matuz B, Liva G, Barrios R, Mata-Calvo R, Giggenbach D. Digital modulation and coding for satellite optical feeder links. In: Advanced Satellite Multimedia Systems Conference and the 13th Signal Processing for Space Communications Workshop (ASMS/SPSC), 2014 7th; 2014; Livorno, Italy:150-157.

10. Barrios Ricardo, Mata-Calvo Ramon, Giggenbach Dirk. RivOLi-TN1: review of RoFSO techniques for optical GEO feeder links; 2015.

11. Gharanjik A, Shankar B, Arapoglou P-D, Ottersten B. Multiple gateway transmit diversity in Q/V band feeder links. IEEE Trans Commun. 2015;63(3): 916-926.

12. Kaushal H, Kaddoum G. Optical communication in space: challenges and mitigation techniques. IEEE Commun Surveys Tutorials. 2016;19:57-96.

13. Lyras NK, Kourogiorgas Cl, Panagopoulos AD. Cloud attenuation statistics prediction from Ka-band to optical frequencies: integrated liquid water content field synthesizer. IEEE Trans Antennas Propag. 2017;65(1):319-328. http://ieeexplore.iee.org/document/7747494/

14. Lyras NK, Efrem CN, Kourogiorgas Cl, Panagopoulos AD. Optimum monthly based selection of ground stations for optical satellite networks. IEEE Commun Lett. 2018;22(6):1192-1195.

15. Perlot N, Perdigues-Armengol J. Model-oriented availability analysis of optical GEO-ground links. Free-Space Laser Communication Technologies XXIV, Bellingham, Washington USA: International Society for Optics and Photonics; 2012:82460P. http://proceedings.spiedigitallibrary.org/proceeding. aspx?doi=10.1117/12.908706

16. Sanchez Net M, del Portillo I, Crawley E, Cameron B. Approximation methods for estimating the availability of optical ground networks. J Opt Commun Netw. 2016;8(10):800. https://www.osapublishing.org/abstract.cfm?URI=jocn-8-10-800

17. Perlot N, Dreischer T, Weinert CM, Perdigues J. Optical GEO feeder link design. In: Future Network Mobile Summit (FutureNetw), 2012; 2012; Berlin, Germany:1-8.

18. Dimitrov S, Barrios R, Matuz B, Liva G, Mata-Calvo R, Giggenbach D. Digital modulation and coding for satellite optical feeder links with pre-distortion adaptive optics. Int J Sat Commun Netw. 2016;34(5):625-644. http://onlinelibrary.wiley.com/doi/10.1002/sat.1163/abstract

19. Andrews LC. Strehl ratio and scintillation theory for uplink Gaussian-beam waves: beam wander effects. Optical Eng. 2006;45(7):76001. http:// opticalengineering.spiedigitallibrary.org/article.aspx?doi=10.1117/1.2219470

20. Andrews LC, Phillips RL. Laser Beam Propagation through Random Media, Vol.Bellingham, Washington USA: PM152: SPIE; 2005.

21. Dios F, Rubio JA, Rodríguez A, Comerón A. Scintillation and beam-wander analysis in an optical ground station-satellite uplink. Appl Opt. 2004;43(19): 3866. https://www.osapublishing.org/abstract.cfm?URI=ao-43-19-3866

22. Degnan JJ. Millimeter accuracy satellite laser ranging: a review. In: Smith DE, Turcotte DL, eds. Geodynamics Series, Vol. 25. Washington, D. C.: American Geophysical Union; 1993:133-162. http://www.agu.org/books/gd/v025/GD025p0133/GD025p0133.shtml

23. Lyras NK, Efrem CN, Kourogiorgas Cl, Panagopoulos AD, Arapoglou P-D. Optimizing the Ground Network of Optical MEO Satellite Communication Systems. IEEE Syst J. 2019;1-9. https://doi.org/10.1109/jsyst.2019.2945838

24. Lyras NK, Efrem CN, Kourogiorgas Cl, Panagopoulos AD. Medium earth orbit optical satellite communication networks: ground terminals selection optimization based on the cloud-free line-of-sight statistics. Int J Sat Commun Netw. 2019;37(4):370-384. https://onlinelibrary.wiley.com/doi/abs/10. 1002/sat.1289

25. Lyras NK, Kourogiorgas Cl, Panagopoulos AD. Cloud free line of sight prediction modeling for optical satellite communication networks. IEEE Commun Lett. 2017;21(7):1537-1540.

26. Giggenbach D, Lutz E, Poliak J, Mata-Calvo R, Fuchs C. A high-throughput satellite system for serving whole Europe with fast internet service, employing optical feeder links. In: Proceedings of Broadband Coverage in Germany. 9th ITG Symposium; 2015; Berlin, Germany:1-7.

27. Mesleh R, Elgala H, Haas H. Optical spatial modulation. IEEE/OSA J Opt Commun Netw. 2011;3(3):234-244.

28. Ozbilgin T, Koca M. Optical spatial modulation over atmospheric turbulence channels. J Lightw Technol. 2015;33(11):2313-2323.

29. Safari M, Uysal M. Do we really need OSTBCS for free-space optical communication with direct detection? IEEE Trans Wirel Commun. 2008;7(11): 4445-4448.

30. Yang G, Khalighi M-A, Virieux T, Bourennane S, Ghassemlooy Z. Contrasting space-time schemes for MIMO FSO systems with non-coherent modulation. In: 2012 International Workshop on Optical Wireless Communications (IWOW); 2012; Pisa, Italy:1-3.

31. Mata Calvo R, Becker P, Giggenbach D, et al. Transmitter diversity verification on ARTEMIS geostationary satellite; 2014:897104. http://proceedings. spiedigitallibrary.org/proceeding.aspx?doi=10.1117/12.2036554

32. Roy B, Poulenard S, Dimitrov S, et al. Optical feeder links for high throughput satellites. In: 2015 IEEE International Conference on Space Optical Systems and Applications (ICSOS). IEEE; 2015; New Orleans, LA, USA:1-6. http://ieeexplore.ieee.org/document/7425074/

33. Simon MK, Vilnrotter VA. Alamouti-type space-time coding for free-space optical communication with direct detection. IEEE Trans Wirel Commun. 2005;4(1):35-39.

34. Park J, Lee E, Yoon G. Average bit-error rate of the alamouti scheme in gamma-gamma fading channels. IEEE Photonics Technol Lett. 2011;23(4): 269-271.

35. Song X, Cheng J. Alamouti-type STBC for subcarrier intensity modulated wireless optical communications. In: 2012 IEEE Global Communications Conference (GLOBECOM); 2012; Anaheim, CA, USA:2936-2940.

36. Ren T-P, Yuen C, Guan YL, Tang G-S. High-order intensity modulations for OSTBC in free-space optical MIMO communications. IEEE Wirel Commun Lett. 2013;2(6):607-610. 
37. Bhatnagar MR, Anees S. On the performance of alamouti scheme in gamma-gamma fading FSO links with pointing errors. IEEE Wirel Commun Lett. 2015;4(1):94-97.

38. Mengali A, Kayhan F, Mysore R BS, Ottersten B. Exploiting diversity in future generation satellite systems with optical feeder links. In: 34th AIAA International Communications Satellite Systems Conference. Cleveland, OH: American Institute of Aeronautics and Astronautics; 2016:5768. https:// doi.org/10.2514/6.2016-5768

39. lizuka K. Elements of Photonics, Vol. 2. Hoboken, New Jersey, United States: Wiley; 2002.

40. Liolis K. ONSET (optical feeder links study for satellite networks) final report. Technical Report ESA Contract No: 4000113462/15/NL/NDe, Report No: ONSET-SPC-TCO-ESA-0053, Version: 2.0.0, Noordwijk, The Netherlands, ESA; 2017.

41. Second generation framing structure, channel coding and modulation systems for broadcasting, interactive services, news gathering and other broadband satellite applications; part 2: DVB-S2 extensions (DVB-S2x). Technical Report EN 302 307-2 V1.1.1, ETSI; 2014. http://www.etsi.org/deliver/ etsi_en/302300_302399/30230702/01.01.01_20/en_30230702v010101a.pdf

42. Ultra-fast $100 \mathrm{GHz}$ photodetector: XPDV412xr. Technical Report, Finisar; 2014.

43. Altera 5sgxma High Bandwidth FPGA. http://www.edn.com/electronics-blogs/out-of-this-world-design/4440307/Introducing-the-world-s-first28nm-semiconductor-for-space

44. Second generation framing structure, channel coding and modulation systems for broadcasting, interactive services, news gathering and other broadband satellite applications; part 1 (DVB-S2). Technical Report EN 302 307-1 V1.4.1, ETSI; 2014. http://www.etsi.org/deliver/etsi_en/302300_ 302399/30230701/01.04.01_60/en_30230701v010401p.pdf

45. Seimetz M. High-Order Modulation for Optical Fiber Transmission, No. 143 in Springer series in optical sciences. Berlin ; New York: Springer; 2009. OCLC: ocn297148328.

46. Li Jia, Liu JohnQ, Taylor DP. Optical communication using subcarrier PSK intensity modulation through atmospheric turbulence channels. IEEE Trans Commun. 2007;55(8):1598-1606.

47. Ghassemlooy Z, Popoola WO, Gao S, Allen JIH, Leitgeb E. Free-space optical communication employing subcarrier modulation and spatial diversity in atmospheric turbulence channel. IET Optoelectron. 2008;2(1):16-23. http://digital-library.theiet.org/content/journals/10.1049/iet-opt_ 20070030

48. Popoola WO, Ghassemlooy Z. BPSK subcarrier intensity modulated free-space optical communications in atmospheric turbulence. J Lightwave Technol. 2009;27(8):967-973.

49. Samimi H. Optical communication using subcarrier intensity modulation through generalized turbulence channels. J Optical Commun Netw. 2012;4(5): 378. https://www.osapublishing.org/abstract.cfm?URI=jocn-4-5-378

50. SpectralCalc Tool. http://www.spectralcalc.com/info/about.php

51. ITU-R P.1622 "Prediction methods required for the design of Earth-space systems operating between $20 \mathrm{THz}$ and $375 \mathrm{THz}$ ". Technical Report, Geneva: International Telecommunication Union; 2003.

52. Kourogiorgas C, Panagopoulos AD. A tropospheric scintillation time series synthesizer based on stochastic differential equations. In: Proceedings of 19th Ka and Broadband Communications, Navigation and Earth Observation Conference; 2013; Florence, Italy.

53. ITU-R P.840-6 "Attenuation due to clouds and fog". Technical Report, Geneva: International Telecommunication Union; 2013.

54. Tse D, Viswanath P. Fundamentals of wireless communication. 4. printing with corr. Cambridge: Cambridge Univ. Press; 2008. OCLC: 254965043.

55. LoTKY. Maximum ratio transmission. IEEE Trans Commun. 1999;47(10):1458-1461. http://ieeexplore.iee.org/document/795811/

56. Tarokh V, Jafarkhani H, Calderbank AR. Space-time block codes from orthogonal designs. IEEE Trans Info Theory. 1999;45(5):1456-1467. http:// ieeexplore.iee.org/document/771146/

57. Zhou S, Giannakis GB. Optimal transmitter eigen-beamforming and space-time block coding based on channel mean feedback. IEEE Trans Signal Process. 2002;50(10):2599-2613. http://ieeexplore.ieee.org/document/1033689/

58. Hemmati H. Near-Earth laser communications, No. 143 in Optical science and engineering. Boca Raton, US: CRC Press; 2009. https://doi.org/10.1201/ 9781420015447

59. Bottacchi S. Noise and signal interference in optical fiber transmission systems: an optimum design approach. Chichester, UK: John Wiley \& Sons; 2008. OCLC: ocn231164172. ISBN: 978-0-470-06061-2. https://www.wiley.com/en-us/Noise+and+Signal+Interference+in+Optical+Fiber+Transmission +Systems\%3A+An+Optimum+Design+Approach-p-9780470060612

60. Ishimaru A. Wave propagation and scattering in random media. Oxford: Elsevier Science; 1978. OCLC: 843198196. http://www.123library.org/book details/?id=101145.

61. DVB-S2 Implementation Guidelines. Technical Report TR 102 376-1 V1.2.1, ETSI; 2015. https://www.etsi.org/deliver/etsi_tr/102300_102399/ 10237601/01.02.01_60/tr_10237601v010201p.pdf

62. Recommendation ITUR G.694.1, spectral grids for WDM applications: DWDM frequency grid. Technical Report, International Telecommunication Union (ITU); 2012.

63. Montmerle Bonnefois A, Conan JM, Petit C, et al. Adaptive optics pre-compensation for GEO feeder links: the FEEDELIO experiment, International Conference on Space Optics - ICSO 2018, 111802C (Vol. 11180). Chania, Greece: SPIE; 2019. https://doi.org/10.1117/12.2536003

\section{AUTHOR BIOGRAPHIES}

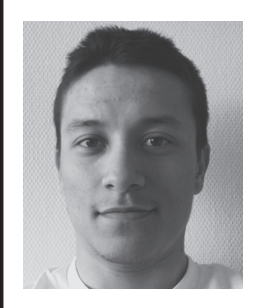

Alberto Mengali obtained his M.Sc (2014) in Telecommunication engineering at the University of Pisa and his PhD (2018) in satellite communications at the University of Luxembourg. Since then he has been working at the European Space Agency on the Telecommunication Systems and Techniques section. His research interests include Active Antennas, Signal Processing, Satellite Systems, Random Access and Optimization. 


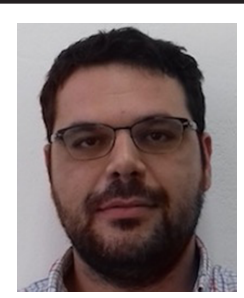

Charilaos I. Kourogiorgas was born in Athens, Greece, on July 6, 1985. He received the Diploma Engineering and the Ph. D. degrees in electrical and computer engineering from the National Technical University of Athens (NTUA), Athens, Greece, in 2009 and May 2015, respectively. From October 2009 to June 2011, he was with the Department of Electromagnetism and Radar at Office National d'Études et Recherches Aérospatiales(ONERA), Toulouse, France. From January to October 2016, he served at the CyberSecurity unit of the Hellenic Army IT Support Center, Athens, Greece. From April 2017 until March 2019, he was with RAL Space as a Marie Sklodowska-Curie Fellow, working on the application of Artificial Intelligence for physical layer techniques and resource allocation. He has authored/coauthored more than 100 papers in international refereed journals and conferences. His research interests include channel modeling for satellite and terrestrial communication systems, the evaluation of system's performance and the application of Artificial Intelligence methods for satellite communication systems. Dr. Kourogiorgas is a Marie Sklodowska Curie Fellow, member of Technical Chamber of Greece. He was the recipient of the Chorafas Scholarship for his Ph.D. studies and the URSI Young Scientist Award for 2014.

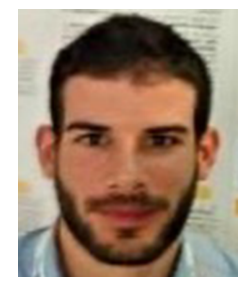

Dr. Nikolaos K. Lyras was born in Kalamata, Greece in 1991. He received the Diploma degree in Electrical and Computer Engineering and the Dr. Engineering Degree from the National Technical University of Athens (NTUA), Athens, Greece, in February 2015 and July 2019, respectively. His PhD Thesis concerned the system level engineering of optical satellite communication systems. He has been involved in 7 European Space Agency and 2 European Union funded research projects. Nikolaos has published more than 30 contributions in international refereed journals and conferences. He has been awarded with George \& Viktoria Karelia Foundation Scholarship for his PhD studies, in 2016. His research interests include optical communication systems design, system level engineering for wireless and satellite communication networks, free-space optics in telecoms and data analysis among others.

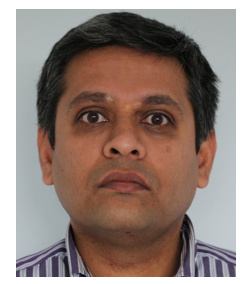

Bhavani Shankar M. R. received Masters and Ph. D in Electrical Communication Engineering from Indian Institute of Science, Bangalore in 2000 and 2007 respectively. He was a Post Doc at the ACCESS Linnaeus Centre, Signal Processing Lab, Royal Institute of Technology (KTH), Sweden from 2007 to September 2009. He joined SnT in October 2009 as a Research Associate and is currently a Research Scientist at SnT. He was with Beceem Communications, Bangalore from 2006 to 2007 as a Staff Design Engineer working on Physical Layer algorithms for WiMAX compliant chipsets. He was a visiting student at the Communication Theory Group, ETH Zurich, headed by Prof. Helmut Bölcskei during 2004. Prior to joining Ph. D, he worked on Audio Coding algorithms in Sasken Communications, Bangalore as a Design Engineer from 2000 to 2001. His research interests include Design and Optimization of MIMO Communication Systems, Automotive Radar and Array Processing, polynomial signal processing, Satellite communication systems, Resource Allocation, Game Theory and Fast Algorithms for Structured Matrices. He is currently on the Executive Committee of the IEEE Benelux joint chapter on communications and vehicular technology and serves as handling editor for Elsevier Signal Processing. He was a co-recipient of the 2014 Distinguished Contributions to Satellite Communications Award, from the Satellite and Space Communications Technical Committee of the IEEE Communications Society.

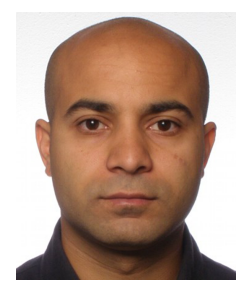

Farbod Kayhan received the B.Sc degree in Mathematics from S.B. University of Kerman in 1999, the M.Sc degree in Modelling and Simulation of Complex Realities from International Center for Theoretical Physics (ICTP) in 2003, and the Ph.D. degree in electrical engineering and telecommunications from Politecnico di Torino in 2007. He has spent one year postdoctoral position at Department of Systems Science of Kyoto University from 2007 to 2008 . He has been a research assistant at Politecnico di Torino from 2009 to 2015 . He is currently a research associate with the SIGCOM group in the Interdisciplinary Centre for Security, Reliability and Trust, University of Luxembourg. His main research interests include modern coding theory and combinatorial optimization with particular emphasis on iterative channel and source coding and constellation space optimization, signal processing and satellite communication systems.

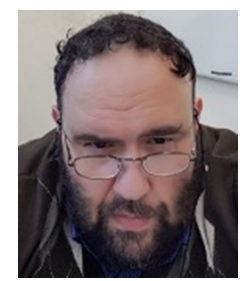

Athanasios D. Panagopoulos (S'98, M'02, SM '09): was born in Athens, Greece on January 26, 1975. He received the Diploma Degree in Electrical and Computer Engineering (summa cum laude) and the Dr. Engineering Degree from National Technical University of Athens (NTUA) in July 1997 and in April 2002 now Currently he is Associate Professor in the School of Electrical and Computer Engineering in NTUA (since May 2017). He has published more than 170 papers in International Journals and IEEE Transactions and more than 215 papers in conference proceedings. He has also published more than 35 Book Chapters in International Books. He is the recipient of URSI General Assembly Young Scientist Award in 2002 and 2005. He is co-recipient of the Best Paper Awards in IEEE RAWCON 2006 and IEEE ISWCS 2015. His research interests include radio communication systems design, wireless and satellite communications networks and mobile computing systems. He has participated to ITU-R and to ETSI Study Groups, and he is member of Technical Chamber of Greece and Senior Member of IEEE. He was Chairman of the IEEE Greek Communication Chapter for 8 years. He has led as Principal Investigator many R\&D programs 
funded by EU and European Space Agency. Finally, he has served on the Editorial boards in Elsevier Physical Communication, in IEEE Transactions on Antennas and Propagation and in IEEE Communication Letters.

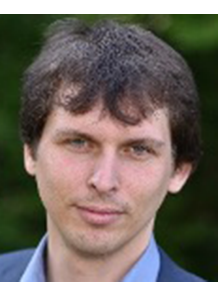

Thomas Bäumer is a Systems Engineer at SES S.A., Luxembourg, managing technology innovation projects on satellite communications. He has technical and managerial experience with ESA, International and National R\&D\&I projects with focus on satellite communications. He received a diploma in mathematics (Dipl.-Math.) with minor in electrical engineering from the University of Ulm.

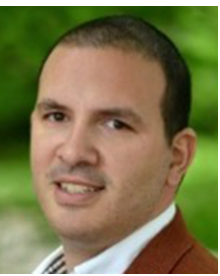

Konstantinos Liolis is Senior Systems Engineer at SES S.A., Luxembourg, managing technology innovation projects on satellite communications. Previously, he was employed as R\&D Project Manager at Space Hellas S.A., Greece; Communication Systems Engineer at the European Space Agency, Research and Technology Centre (ESA/ESTEC), The Netherlands; and Research Assistant at the California Institute for Telecommunications and Information Technology (Cal-IT2), San Diego, USA. He has over 15 years of experience, both technical and managerial, in more than 40 contracted EU, ESA and National R\&D\&I projects in ICT with major focus on satellite communications. He has published more than 70 scientific papers in international peer-reviewed journals, conference proceedings and book chapters, in areas mainly related to satellite communications. He has also numerous contributions to the ETSI, 3GPP, DVB and ITU-R international standardization bodies. He received the Dipl.-Eng. and PhD degrees in Electrical and Computer Engineering from the National Technical University of Athens (NTUA), Greece; and the MSc degree in Electrical Engineering from the University of California at San Diego (UCSD), USA. He is PMI PMP ${ }^{\circledR}$ certified project manager and Member of the Technical Chamber of Greece (TEE). He received a Best Student Paper Award in IEEE RAWCON2006. He currently serves the Editorial Board of the Wiley's International Journal of Satellite Communications and Networking.

How to cite this article: Mengali A, Kourogiorgas CI, Lyras NK, et al. Ground-to-GEO optical feeder links for very high throughput satellite networks: Accent on diversity techniques. Int J Satell Commun Network. 2020;1-22. https://doi.org/10.1002/sat.1372 